\title{
Sur les singularités des écoulements plans et permanents des nappes souterraines pesantes
}

\author{
Singularities of plane \\ and permanent gravity flow of ground water
}

\section{J. KRAVTCHENKO,}

PROFESSEUR a LA FACULTÉ DES SCIENCES DE GRENOBLE
PAR

\author{
(x. SAUVAGE DE SAINT-MARC,
}

DIRECTEUR ADJOINT

DU LABORATOIRE DAUPHINOIS D'HYDRAULIQUE

\author{
ET M. BORELI \\ INGÉNIEUR-DOCTEUR \\ INSTITUT HYDROTECHNIQUE, BELGRADE
}

\begin{abstract}
Le présent mémoire est consacré à l'étude des singularités que peut présenter un écoulement plan et permanent d'un liquide pesant en milieu poreux, isotrope, et homogène. Le plan du mouvement est vertical; le liquide est soumis à l'action de la pesanteur seule. La discussion prêsentée est essentiellement locale; elle suppose connue a priori, dans le plan de la vitesse complexe, l'image conforme du voisinage de la singularité. Moyennant cette donnée, le problème peut être complètement résolu; les règles propres à fixer le choix de ce voisinage sont laissées de côté. L'importance de cette étude dans l'art de l'ingénieur est mise en relief.

Pour simplifier et alléger l'exposition, quelques résultats théoriques de Poloubarinova-Kotchina sont supposés connus; une démonstration élémentaire en sera publiée ailleurs.
\end{abstract}

\begin{abstract}
The present note is devoted to the study of singularities that can occur in a plane steady gravity flow of liquid in a porous medium which is isotropic and homogeneous. The plane of movement is vertical; the liquid is subject to the action of gravity only. The discussion presented is for localised areas; it supposes known, in the complex velocity plane, the conformal pattern in the neighbourhood of the singularity. Assuming this given, the problem can be solved completely; the rules for fixing the choice of this neighbourhood are not considered. The importance of this study in engineering practice is illustruled.

To simplify and lighten the argument, "t few theoretical results of poloubarinovaKotchina are taken fort granted; an elementary demonstration of them will be published elsewhere.
\end{abstract}

\section{INTRODUCTION}

Considérons, dans un plan vertical, un écoulement plan, permanent, à potentiel, d'un liquide pesant en milieu poreux, homogène, isotrope. Le domaine empli par le liquide en mouvement est limité par des segments rectilignes (par exemple : parois et fond imperméables, frontières perméables du milieu poreux, lignes de suintement, etc.), et des lignes libres. On se propose d'étudier ici les singularités que peut présenter un tel écoulement en un point frontière commun à deux éléments de frontière de natures différentes : par exemple, en un point commun à une ligne libre et à une surface de suintement.

La méthode employée pour résoudre ce pro- blème est une variante de celle de PoloubarinovaKotchina. Quelques compléments originaux donnent le moyen de pousser l'étude du voisinage de la singularité jusqu'aux éléments du second ordre et de préciser, en particulier, l'allure de la courbure d'une ligne libre en son point de raccordement avec un autre élément frontière. On complète ainsi les résultats classiques de CASAGRANDE relatifs à l'existence et à l'orientation limite des tangentes à la ligne libre.

Nos conclusions paraissent présenter quelque intérêt pour l'ingénieur. D'une part, en effet, la connaissance à priori des singularités est essentielle pour construire un écoulement par des pro- 
cédés approchés - les seuls susceptibles de rendre service dans l'étude théorique d'un projet d'ouvrage. D'autre part, on verra que ces singularités sont loin d'avoir une allure simple qui en permettrait une détermination élémentaire. Il nous a, dès lors, semblé opportun de présenter un exposé synthétique de la théorie des singularités. abordée trop souvent jusqu'ici par les seuls moyens qu'offre l'intuition. Mais si le recours à l'intuition permet souvent d'abréger les calculs, il n'est pas dépourvu de risques. Nous avons donc volontairement présenté nos conclusions sous une forme qu'exige la rigueur, mais dont nous sommes les premiers à regretter l'abs- traction. Nous illustrons la théoric générale en discutant à fond deux exemples; nous utilisons une méthode uniforme, renonçant à l'emploi des remarques géométriques propres à abréger les calculs. Nous espérons ainsi mettre en évidence la puissance et la généralité des méthodes de Poloubarinova-Kotchina.

Mais il est bien entendu que le praticien pourra -- et même devra toujours - simplifier les calculs en utilisant de judicieux artifices. Nous comptons, dans une prochaine publication, présenter un autre exemple de discussion d'une singularité, plus conforme à l'esprit «ingénieur ».

\section{CHAPITRE PREMIER}

\section{ÉNONCE ET HISTORIQUE DU PROBLEME}

1. - Dans un plan vertical, considérons un système d'axes rectangulaires $O x y$, d'origine $O$, arbitraire, et dont l'axe $O y$ est vertical ascendant. Nous noterons : $g$ l'accélération de la pesanteur; $z=x+i y$, la variable complexe du plan $\mathrm{O} x y$; D, un domaine simplement connexe de ce plan; C, la frontière de D que nous supposons simple, pour simplifier - en excluant ainsi le cas, utile dans la pratique, où $\mathrm{D}$ comprendrait des coupures.

Envisageons dans $D$ un écoulement permanent, plan, d'eau souterraine, de densité $\rho$, soumise à l'action de la pesanteur seule. Nous admettrons que D est constitué par un milieu poreux homogène et isotrope, caractérisé, au point de vue qui nous occupe, par sa constante de Darcy $k$. Rappelons rapidement quelques propriétés classiquies de ces régimes.

Le champ des vitesses $\overrightarrow{\mathrm{V}}(x, y)$ dérive du potentiel uniforme $\varphi(x, y)$; il existe une fonction de courant $\psi(x, y)$; $\varphi$ et $\psi$ sont deux fonctions harmoniques conjuguées, régulières dans $\mathrm{D}$. Le potentiel complexe $f(z)$ est une fonction analytique de $z$, holomorphe dans $\mathrm{D}$, définie par:

$$
f(z)=\varphi+i \psi \text {. }
$$

La vitesse complexe :

$$
\Omega(z)=\frac{d f}{d z}=u-i v,
$$

où $u$ et $v$ sont les composantes du vecteur $\vec{v}=\overrightarrow{\operatorname{grad}} \varphi$ suivant les axes, est donc également une fonction holomorphe en chaque point de $D$. On notera que $u+i v$ n'est pas une fonction monogène de $z$ dans $D$.
Il y a souvent intérêt à introduire la fonction de Levi-Crvita :

$$
\omega(z)=\theta+i \tau
$$

définie par :

$$
\Omega(z)=e^{-i \omega}
$$

On a donc : $|\mathrm{V}|=e^{\tau} ; \arg \overrightarrow{\mathrm{V}}=\theta$. L'usage de $\omega(z)$ se recommande particulièrement dans l'étude des voisinages des parois solides imperméables limitant $D$, particulièrement des points anguleux de telles parois. Mais pour simplifier, nous éliminerons ces difficultés classiques de notre étude et, dans la suite, nous n'aurons pas à utiliser le plan $\omega$.

Les correspondances $f=f(z)$ et $\Omega=\Omega(z)$ réalisent l'application conforme de $\mathrm{D}$ sur les domainés, simplement connexes, en général, des plans $f$ et $\Omega$ que nous appellerons $\Delta$ et $\delta$ respectivement et dont nous noterons respectivement $\Gamma$ et $\gamma$ les frontières. Nous désignerons par $p=p(x, y)$ la pression hydrostatique au point $x, y$ de $\mathrm{D}$.

La loi de DARcy, dont nous avons admis la validité dans toute l'étendue de $\mathrm{D}$, donne :

$$
\vec{V}=-k \overrightarrow{\operatorname{grad}} h .
$$

On a posé :

$$
h=\frac{p}{\rho g}+y
$$

Enfin, l'équation de Bernoullr s'applique et se réduit ici à :

$$
\frac{\varphi}{k}+\frac{p}{\rho g}+y=\mathrm{C}^{\mathrm{te}} \quad \text { dans } \mathrm{D}
$$


2. - Portons notre attention sur la frontière $C$ de D. Dans les cas les plus courants, C comprend d'abord un certain nombre d'éléments donnés, à priori (sauf réserve spécifiée ci-après à propos des lignes de suintement), et appartenant à l'un des types suivants :

1. Les parois imperméables, que nous désignerons par $\mathrm{C}_{1}$;

2. Les ares de courbes $\mathrm{C}_{2}$, lignes de séparation (perméables au liquide) de $\mathrm{D}$ avec des bassins remplis d'eau, par où le liquide pénètre dans le milieu poreux D - ou sort de ce domaine. Nous rangerons encorc sous cette rubrique les lignes-drains, le long desquelles les eaux souterraines sont, soit aspirées vers l'extérieur de $D$, soit refoulćes vers l'intérieur de D sous l'effet d'une sous-pression ou d'une surpression convenable;

3. Les lignes de suintement $\mathrm{C}_{33}$; le liquide sort de $D$ le long de ces lignes à l'air libre et s'écoule le long d'elles. Il faut noter -point qui sera précisé ultérieurement qu'une des extrémités des lignes $\mathrm{C}_{1}, \mathrm{C}_{2}$ ou $\mathrm{C}_{3}$ peut n'être pas connue, à priori.

Enfin, C peut contenir encore des surfaces libres $\mathrm{C}_{4}$ qui sont, par définition, des lignes de courant à pression constante; les éléments de $\mathrm{C}$ du type $C_{4}$ sont de forme, à priori, inconnue; toutefois, la position de l'une des extrémités de $C_{4}$ est une donnée.

Bien entendu, la frontière $C$ peut comprendre plusieurs éléments de chacun des types $\mathrm{C}_{n}$ $(n=1,2,3,4)$.

Pour simplifier notre exposé, nous admettrons que les éléments $C_{1}, C_{2}, C_{3}$ de $C_{4}$, donnés à priori, sont constitués par des segments rectilignes. C'est là un cas qui intéresse tout spécialement l'ingénieur. Mais l'extension aux cas où les $\mathrm{C}_{1}, \mathrm{C}_{2}, \mathrm{C}_{3}$ seraient des arcs de courbes assez régulières est aisée.

Rappelons rapidement les conditions frontières que vérifient les quantités $\varphi, \downarrow, x, y$, le long de $\mathrm{C}$. Comme les $\mathrm{C}_{1}, \mathrm{C}_{2}, \mathrm{C}_{3}$ sont des segments de droite donnés à priori, on aura tout d'abord sur $\mathrm{C}_{n}$ :

$$
a_{n} x+b_{n} y+c_{n}=0,(n=1,2,3)
$$

où $a_{n}, b_{n}, c_{n}$, sont des constantes données. De plus :

1. $C_{1}$ est toujours une ligne de courant; on a done :

$$
\psi=\mathrm{C}^{\mathrm{t} *} \quad \text { sur } \mathrm{C}_{1}
$$

2. C.. est loujours une équipotentielle; done :

$$
\varphi=\mathrm{C}^{\mathrm{t}+\mathrm{s}} \quad \text { sur } \mathrm{C}_{2}
$$

3. $\mathrm{C}_{3}$ est une ligne à pression constante, égale, au surplus, à la pression atmosphérique.

Le théorème de Bernoulli donne alors:

$$
\varphi+k y=\mathrm{C}^{\text {te }} \quad \text { sur } \mathrm{C}_{3}
$$

De la définition mème de $C_{4}$, il résulte que le long de cet élément de $C$, les conditions (2) et (4) seront toujours vérifiées. Par contre, la forme géométrique de $\mathrm{C}_{4}$ est, à priori, inconnue.

En résumé, le long de chaque élément de $C$, les quatre variables $\varphi, \psi, x, y$, sont liées par deux relations linéaires à coefficients constants, connus à priori; mais il faut souligner que les constantes qui figurent aux seconds membres de (2), (3) et (4) ne sont, en général, pas données.

Nous noterons $\Gamma_{n}$ et $\gamma_{n}$, les éléments de $\Gamma$ el de $\gamma$, images de $\mathrm{C}_{n}(n=1,2,3,4)$ dans les plans $f$ et $\Omega$ respectivement. D'après (2) et (3), $\Gamma_{1}, \Gamma_{2}$ et $\Gamma_{4}$ sont des segments de droite parallèles aux axes du plan $f$; par contre, $\Gamma_{3}$ est une ligne de forme à priori inconnue.

On précisera la forme géométrique de $\gamma_{n}$ en utilisant le raisonnement ci-après, à la fois élémentaire et classique. Différencions (1), (2), (3) et (4); il vient, eu égard à la définition de de $\psi$ :

$$
\begin{array}{cl}
a_{n} d x+b_{n} d y & =0 \\
v d x-\| d y & =0 \\
u d x+v d y & =0 \\
u d x+(v+k) d y=0
\end{array}
$$

On obtient donc la relation entre $u$ et $v$ le long de $\gamma_{n}$ (c'est-à-dire l'équation cartésienne de la courbe symétrique par rapport à l'axe O'u de $\gamma_{n}$ ), en éliminant $d x$ et $d y$ entre deux des relations $\left(1^{\prime}\right),\left(2^{\prime}\right)$, (3') et $\left(4^{\prime}\right)-$ qui sont valables sur $\gamma_{n}$ moyennant le changement de $v$ en $-v$ en vertu de la définition même de cet élément frontière $\left(^{*}\right)$. Ainsi, une $\gamma_{1}$ [caractérisée par (1') el $\left.\left(2^{\prime}\right)\right]$, a pour équation :

$$
a_{1} u-b_{1} v=0
$$

De même, on aura :

$$
\begin{aligned}
a_{2} v+b_{2} u & =0 \text { pour } \gamma_{2} \\
a_{3}(k-v)-b_{3} u & =0 \text { pour } \gamma_{3} \\
u^{2}+v^{2}-k v & =0 \text { pour } \gamma_{4}
\end{aligned}
$$

Ainsi, $\gamma_{1}, \gamma_{2}$ el $\gamma_{3}$ sont des segments rectilignes dont une des extrémités peut n'être pas connue à priori; $\gamma_{4}$ est un arc de cercle (6). Mais il est essentiel de noter que les équations des $\gamma_{n}(n=1$,

(*) On se rappellera que $\Omega=u-i v$; il s'ensuit que le vecteur $\vec{v}$ et le vecteur de composantes $u$ et $-v$ sont symétriques par rapport à $\mathrm{O} a$. 
$2,3,4)$ ne font intervenir que les constantes $a_{n}, b_{n}$ et $k$ qui sont des données. Il en résulte que la connaissance à priori de la nature de $\mathrm{C}$ c'est-à-dire de la nature et de l'ordre de succession des différents éléments constitutifs $\mathrm{C}_{n}(n=1$, $2,3,4)$ - suffit pour déterminer la forme générale du polygone mixtiligne $\gamma$, formé de segments de droites et d'arcs de cercle; en particulier, les angles de $\gamma$ sont des données, mais la longueur des $\gamma_{n}$ peut être une inconnue du problème.

Nous pouvons maintenant faire comprendre la raison pour laquelle l'une des extrémités des éléments de $\mathrm{C}$ ou de $\Gamma$, de supports donnés, peut être, à priori, inconnue. Rappelons que $\mathrm{C}_{4}$ et $\mathrm{\Gamma}_{3}$ sont des éléments inconnus, à priori, dans leurs plans respectifs $z$ et $f$. Si C, par exemple, contient une $\mathrm{C}_{4}$, l'une de ses extrémités est une donnée, mais la position du point où cette ligne se raccorde à un élément contigu $\mathrm{C}_{1}$ ou $\mathrm{C}_{3}$ peut n'être pas connue (bien que la droite-support de l'un de ces segments soit une donnée). Dans le plan $f$, l'indétermination peut être plus grande encore, en ce qui concerne le raccord de $\Gamma_{3}$ avec I'élément contigu $\Gamma_{2}$ par exemple. Car, ici, la droite-support de $\Gamma_{2}$, d'après ce qu'on a vu, n'est déterminée qu'à une translation près.

3. - La correspondance $f=f(z)$, holomorphe dans D, l'est aussi - comme son inverse $z=z(f)$ - en chaque point intérieur de l'élément $\mathrm{C}_{n}(n=1,2,3,4)$ - ou de son image $\Gamma_{n}$ dans le plan $f$.

En effet, l'analyse présentée au précédent paragraphe prouve que, quel que soit $\mathrm{C}_{n}$, deux, au moins, des trois éléments de frontières homologues $C_{n}, \gamma_{n}$ et $\Gamma_{n}$ sont soit des segments de droite, soit des arcs de cercle. Le théorème classique de Schwarz - conceernant la correspondance entre frontières analytiques dans la représentation conforme - entraîne alors l'holomorphie de l'une, au moins, des trois correspondances : $\Omega=\Omega(z), \Omega=\Omega(f) ; f=f(z)$ (ou de leurs inverses) à l'intérieur de $\mathrm{C}_{n}$ ou de ses images dans $f$ ou $\Omega$. Le résultat est donc justifié si $\mathrm{C}_{n}$ et $\Gamma_{n}$ sont des segments de droite. Si, maintenant, le raisonnement précédent prouve, par exemple, l'holomorphie de $\Omega=\Omega(f)$ sur $\mathrm{C}_{4}$, une simple intégration étend la conclusion à $f=f(z)$.

Les raisonnements élémentaires et classiques qui précèdent montrent bien que $f=f(z)$ ne peut présenter de singularité qu'en un point $z=z_{n m}$, commun à deux éléments $\mathrm{C}_{n}$ et $\mathrm{C}_{m}$ de $\mathrm{C}(n \neq m ; n, m=1,2,3,4)$; nous nommerons sommet de $\mathrm{C}$ un tel point $z_{n m}$.

Le but du présent mémoire est de préciser la singularité de $f(z)$ au point $z_{n m}$ pour toute combinaison d'indices $n$ et $m, n \neq m$.

A la vérité, la solution de ce problème est élémentaire dans la plupart des cas que nous aurons à examiner. On sait, en effet, que l'allure de $f(z)$ en un sommet $z_{n m}$ de $\mathrm{C}-$ ou, ce qui revient au même, l'allure de $\Omega(f)$ ou de $\Omega(z)$ au point homologue de $z_{n m}$ - ne dépend que des propriétés géométriques locales de l'un des couples $(C, \Gamma)$, $(\gamma, C),(\Gamma, \gamma)$ dans le voisinage $d u$ sommet considéré. Or, d'après les conclusions du paragraphe 2, $\gamma$ est parfaitement déterminé dans le voisinage de chacun de ses sommets; il en est de même de l'un, au moins des polygones mixtilignes $\Gamma$ et $\mathrm{C}$ - sauf au point $z_{34}$, commun à $\mathrm{C}_{3}$ et $\mathrm{C}_{4}$. Ce cas exceptionnel écarté, le problème posé revient donc à étudier la correspondance conforme entre deux domaines dans le voisinage des deux points anguleux homologues de leurs frontières; chaque point anguleux étant constitué par l'intersection de deux segments de droite ou d'un segment de droite et d'un arc d'un cercle donné (tangents éventuellement entre eux). Les méthodes élémentaires permettent alors de conclure; mais leur application exige parfois des calculs fastidieux.

Nous nous proposons de présenter une discussion absolument générale et rapide des singularités de $f(z)$ - englobant, en particulier, le cas exceptionnel mentionné ci-dessus - et, dès lors, propre à satisfaire le technicien.

4. Car le problème qu'on vient d'énumérer est de grande importance pour l'ingénieur. La détermination explicite de $f(z)$ n'est possible que dans un petit nombre de cas, dont on trouvera la nomenclature dans les traités classiques de Muscat [1] $\left(^{*}\right)$ et de Mme Poloubarinova-Kotchina [2]. Il s'ensuit que pour résoudre des problèmes concrets, l'ingénieur est obligé d'avoir recours aux méthodes d'approximation et aux méthodes analogiques (cuves électrolytiques, etc.) dont le rendement et la sécurité sont très améliorés chaque fois que l'on connaît, à priori, les singularités de la fonction inconnne $f(z)$. C'est pourquoi nous avons cru faire œuvre utile en publiant cette mise au point de l'ćtat actuel de la théorie des singularités.

5. Avant d'entrer dans le vif du sujet, faisons un bref historique de la question et présentons un résumé des chapitres ultérieurs de ce travail.

Il est curieux de constater que, malgré son importance pratique, l'étude des singularités de $f(z)$ n'ait suscité des recherches systématiques que depuis peu de temps. A cet égard, il convient de signaler, en premier lieu, les nombreux travaux russes, spécialement ceux de Mme PoloubaRINOVA-KotchINA, dont on trouvera dans [2], chapitre VII, une copieuse bibliographie. Le grand traité de cet auteur est fondamental. L'essentiel de la théorie y est déjà exposé et un pa-

(*) Les chiffres entre crochets renvoient à la bibliographie qu'on trouvera à la fin de l'article. 
ragraphe entier du chapitre précité est consacré à la discussion des singularités de $f(z)$. Mais, d'une part, la méthode de [2] paraît un peu lourde; d'autre part, sa mise en œuvre n'a pas été poussée assez loin pour discuter quelques problèmes importants, comme, par exemple, l'étude de $\mathrm{C}_{4}$ dans le voisinage de ses extrémités. On trouvera dans la courte note [3] une variante simplifiée des raisonnements de [2] - déjà utilisés dans la récente thèse de Boreli [4] — et quelques compléments propres à combler les la- cunes de [2], signalées plus haut. Un mémoire à paraître [5] donnera la justification rigoureuse de notre méthode, dont nous résumons l'essentiel au chapitre II ci-après; puis au chapitre III, nous appliquerons les procédés de discussion de [2], modifiés par [3] et [4], à l'examen de deux cas typiques.

Signalons qu'on trouvera dans [4] des exemples de l'étude des singularités de $f(z)$ au moyen des procédés élémentaires dont on a décrit au paragraphe 3 le principe.

\section{CHAPITRE II}

\section{ETUDE GÉNÉRALE DES SINGULARITÉS DE $f(z)$}

6. Introduisons la variable complexe auxiliaire: $\zeta=\xi+i \eta$. Soit $z=z(\zeta)$, la fonction analytique, holomorphe dans le demi-plan supérieur $\Sigma: \eta \geqslant 0$ du plan $\zeta$ qui réalise l'application conforme de $D$ sur $\Sigma$. L'image de C sera l'axe réel : $\eta=0$. On peut, sans restreindre la généralité, supposer que le sommet $z_{n m}$ (cf. le paragraphe 3) de C $(n, m=1,2,3,4 ; n \neq m)$ a pour homologue le point $\zeta=0$. Soit alors $\eta=0 ; a \leqslant \xi \leqslant 0$ (ou $\zeta=0,0 \leqslant \xi \leqslant b$ ) l'image dans $\Sigma$ de $\mathrm{C}_{n}$ (ou de $\mathrm{C}_{m}$ ); $a$ et $b$ sont des constantes réelles. On montre immédiatement que $z=z(\zeta)$ est holomorphe sur chacun des segments réels qu'on vient de définir, sauf peut-être en leurs extrémités. Il en résulte que $f=f[z(\zeta)]$ possède la même propriété. II est clair que la connaissance des singularités à l'origine $\zeta=0$ des fonctions :

$$
z=z(\zeta)=x(\xi, n)+i y(\xi, \eta)
$$

et :

$f=f(\zeta)=\varphi[x(\xi, \eta), y(\xi, \eta)]+i \psi\left[x(\xi, \eta), y\left(\xi, \eta_{1}\right)\right]$

suffit à déterminer l'allure de $f=f(z)$ dans le voisinage de $z_{n m}$. Cherchons à expliciter dans le plan $\zeta$ les conditions frontières que vérifient $z(\zeta)$ et $f(\zeta)$.

Au paragraphe 2, on a vu que les quatre fonctions de $\xi, \eta: x, y, \varphi$, et $\psi$, harmoniques dans $\Sigma$ et deux à deux conjuguées, étaient liées sur chaque $\mathrm{C}_{n}$ par deux relations linéaires à coefficients constants. Ơn peut donc écrire, d'une manière générale :

$$
\mathrm{M}_{n p} x+\mathrm{N}_{n p} y+\mathrm{P}_{n p} \stackrel{\varphi}{ }+\mathrm{Q}_{n p} \psi=\mathrm{E}_{n p}
$$

pour : $\eta=0 ; \quad x \leqslant \xi \leqslant 0 ; \quad(p=1,2)$

où les coefficients de $x, y$, $\varphi$ et $\psi$ sont des constantes réelles, données à priori.
Des conditions analogues valent pour le segment $\eta=0,0 \leqslant \xi \leqslant b$; nous les noterons en changeant $n$ en $m$ dans les relations précédentes. Désignons, d'une manière générale, par le symbole $\mathrm{I}_{m} \mathrm{~F}(\zeta)$, la partie imaginaire d'une fonction complexe quelconque $F(\zeta)$. Il est clair que les conditions précédentes peuvent encore s'écrire :

$\mathrm{I}_{m}\left[\left(\mathrm{~N}_{n p}+i \mathrm{M}_{n p}\right) z+\left(\mathrm{Q}_{n p}+i \mathrm{P}_{n p}\right) f\right]=\mathrm{E}_{n p} ; p=1,2$ pour $\eta=0, a \leqslant \xi \leqslant 0$.

Posons alors :

$$
\begin{gathered}
d z / d \zeta=Z(\zeta) ; \quad d f / d \zeta=\mathrm{F}(\zeta) \\
\alpha_{p}=\mathrm{Q}_{n p}+i \mathrm{P}_{n p} ; \quad \beta_{p}=\mathrm{N}_{n p}+i \mathrm{M}_{n p}
\end{gathered}
$$

en différenciant les deux relations précédentes sur le segment image de $\mathrm{C}_{n}$ - ce qui est légitime, puisque $z(\zeta)$ et $f(\zeta)$ sont holomorphes à l'intérieur de $\mathrm{C}_{n}-$ on trouve les relations du type :

$\mathrm{I}_{m}\left(\alpha_{p} \mathrm{~F}+\beta_{n} \mathrm{Z}\right)=0 ; p=1,2 ; \eta=0 ; a \leqslant \xi \leqslant 0$,

où $\alpha_{p}, \beta_{p}$ sont des constantes complexes, données à priori. On aurait, de même, les deux conditions aux limites :

$$
\mathrm{I}_{m}\left(\mathrm{~A}_{p} \mathrm{~F}+\mathrm{B}_{p} \mathrm{Z}\right)=0 ; p=1,2 ; \eta=0 ; 0 \leqslant \xi \leqslant b
$$

où les $A_{p}$ et $B_{p}$ sont des constantes complexes données :

$$
\left.\begin{array}{l}
\mathrm{A}_{p}=\mathrm{Q}_{m p}+i \mathrm{P}_{m p} \\
\mathrm{~B}_{p}=\mathrm{N}_{m p}+i \mathrm{M}_{m p}
\end{array}\right\} p=1,2
$$

Inversement, d'après (7), les fonctions :

$$
\alpha_{p} \mathbf{F}+\beta_{p} \mathbf{Z}=f_{p}(p=1,2)
$$


sont prolongeables analytiquement à travers le segment $\eta=0, a \leqslant \xi \leqslant 0$ si $Z$ et $F$ sont finies et continues sur ce segment. Il en résulte que $\mathrm{Z}(\zeta)$ et $\mathrm{F}(\zeta)$ sont holomorphes sur $\mathrm{C}_{n}$, car dans tous les cas pratiques, on aura :

$$
\left|\begin{array}{ll}
\alpha_{1} & \beta_{1} \\
\chi_{2} & \beta_{2}
\end{array}\right| \neq 0 ;\left|\begin{array}{ll}
A_{1} & B_{1} \\
A_{2} & B_{2}
\end{array}\right| \neq 0 .
$$

On pourra donc résoudre en $Z$ et $F$ les relations $f_{p}=\alpha_{p} \mathrm{~F}+\beta_{p} \mathbf{Z}$.

Le problème posé revient donc à déterminer les singularités des fonctions $Z(\zeta)$ et $F(\zeta)$ pour $\zeta=0$, sachant qu'ellès sont assujetties à vérifier les conditions frontières (7) et $\left(7^{\prime}\right)$.

7. Dans [2], Poloubarinova-Kotchina parvient à la solution en s'inspirant de la théorie des équations différentielles linéaires du type de Fuchs. Nous renvoyons à [5] pour une démonstration élémentaire des résultats essentiels de cet auteur que nous allons simplement énoncer.

On établit d'abord que dans le voisinage de $\zeta=0, F(\zeta)$ et $Z(\zeta)$ sont nécessairement de la forme :

$$
\mathrm{F}(\zeta)=\mathrm{K}_{1} \mathrm{U}_{1}+\mathrm{K}_{2} \mathrm{U}_{2} ; \quad \mathrm{Z}=\mathrm{K}_{3} \mathrm{U}_{1}+\mathrm{K}_{4} \mathrm{U}_{2}
$$

avec :

$$
\mathrm{U}_{1}(\zeta)=\zeta^{\lambda_{1}} u_{1}(\zeta) ; \mathrm{U}_{2}(\zeta)=\zeta^{\lambda} u_{2}(\zeta)
$$

Dans $(9)$, les $K_{j}(j=1,2,3,4)$ sont des constantes complexes, inconnues à priori; $u_{1}(\zeta)$ et $u_{2}(\zeta)$ sont des fonctions holomorphes pour $\zeta=0$, telles que $u_{1}(0)=u_{2}(0)=1 ; \lambda_{1}$ et $\lambda_{2}$ sont des constantes, nécessairement réelles (chaque fois que $\gamma$ est un contour continu dans le voisinage d'un sommet homoloque à $\zeta=0$ ), inconnues à priori. Les exposants $\lambda_{1}$ et $\lambda_{2}$, dits exposants caractéristiques, seront des racines d'une équation (E) que l'on formera ci-après. En général, on aura : $\lambda_{1} \neq \lambda_{2}+r, r$ étant entier. Si $\lambda_{1}-\lambda_{2}=r$, les équations (10) sont à remplacer par les suirantes :

$$
\mathrm{U}_{1}(\zeta)=\zeta^{\lambda} u_{1}(\zeta) ; \mathrm{U}_{2}(\zeta)=\zeta^{\lambda} u_{2}(\zeta) \log \zeta
$$

car, dans les cas pratiques (cf [5]), $r=0$.

Nous poserons :

$$
\begin{gathered}
\mathrm{K}_{j}=\mathrm{K}_{j}^{\prime}+i \mathrm{~K}_{j}^{\prime \prime}=\mathrm{R}_{j} e^{i \pi \Phi_{j}} \quad(j=1,2,3,4) \\
x_{p}=\mathrm{Q}_{n p}+i \mathrm{P}_{n p}=\imath_{1 p} e^{i \pi \mu_{i p}} ; \\
\beta_{p}=\mathrm{N}_{n p}+i \mathrm{M}_{n p}=\rho_{2 p} e^{i \pi \mu_{2 p}} ; \quad(p=1,2) \\
\mathrm{A}_{p}=Q_{m p}+i \mathrm{P}_{m p}=\vartheta_{3 p} e^{i \pi \mu_{3 p}} ; \\
\mathrm{B}_{p}=\mathrm{N}_{m p}+i \mathrm{M}_{m p}=\overbrace{\downarrow p} e^{i \pi \mu_{4 p}} .
\end{gathered}
$$

8. Nous allons utiliser les résultats précédents, que nous considérerons comme connus, pour expliciter $\lambda_{1}$ et $\lambda_{2}$ et pour déterminer les $\mathrm{K}_{j}$. Admettons d'abord que $\lambda_{1}-\lambda_{2} \neq r$; on peut supposer que $\lambda_{1}>\lambda_{2}$. Portons les expressions (9) et (10) de $F$ et de $Z$ dans (7) et (7'), en supposant que $\zeta^{\lambda_{1}}$ et $\zeta^{\lambda_{2}}$ se réduisent à leurs déterminations réelles pour $\zeta=\xi>0$. Alors (7') donne, eu égard à (11), pour $\zeta=\xi>0$ :

$$
\begin{aligned}
& \mathrm{I}_{m}\left(\mathrm{~A}_{p} \mathrm{~F}+\mathrm{B}_{p} \mathrm{Z}\right)=\xi^{\lambda: \mathrm{I}_{m}}\left\{\rho_{3 p} \mathrm{R}_{2} e^{i \pi\left(\Phi_{2}+\mu_{3 p}\right)}+\rho_{4 p} \mathrm{R}_{4} e^{i \pi\left(\Phi_{4}+\mu_{4 p}\right)}+\ldots\right. \\
& +\xi^{\lambda_{1}-\lambda_{2}}\left[\rho_{3 p} \mathbf{R}_{1} e^{i \pi\left(\Phi_{1}+\mu_{3 p}\right)}+\left[\rho_{4 p} \mathbf{R}_{3} e^{i \pi\left(\Phi_{3}+\mu_{3} p\right)} \ldots\right]\right\}=0 ; \quad(p=1,2),
\end{aligned}
$$

où le symbole... désigne des séries entières, absolument convergentes pour $|\xi|$ assez petit. Supprimons le facteur réel $\xi^{\lambda_{2}} \mathrm{du}$ premier membre de (12) et faisons tendre $\xi$ vers zéro dans l'expression entre crochets. Comme $\lim _{\xi \rightarrow 0} \xi^{\lambda_{1}-\lambda_{2}}=0$, il est clair que (12) entraîne :

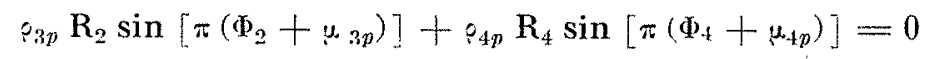

On a supposé que $\lambda_{1}-\lambda_{2}$ n'est pas un entier positif : (12) entraîne encore :

$$
\rho_{3 p} \mathrm{R}_{1} \sin \left[\pi\left(\Phi_{1}+\mu_{3 p}\right)\right]+\rho_{4 p} \mathrm{R}_{3} \sin \left[\pi\left(\Phi_{3}+\mu_{4 p}\right)\right]=0
$$

Le cas où $\lambda_{1}-\lambda_{2}$ est un entier sera étudié au paragraphe 10.

Portons, de même, dans (7), les expressions (9) et (10) de F et de $Z$, en supposant que $\zeta=\xi=|\xi| e^{\pi i}$. Un raisonnement identique au précédent donne les quatre relations :

$$
\begin{aligned}
& \rho_{1 p} R_{2} \sin \left[\pi\left(\Phi_{2}+\mu_{1 p}+\lambda_{2}\right)\right]+\imath_{2 p} R_{4} \sin \left[\pi\left(\Phi_{4}+\mu_{2 p}+\lambda_{2}\right)\right]=0 \\
& \rho_{1 p} R_{1} \sin \left[\pi\left(\Phi_{1}+\mu_{1 p}+\lambda_{1}\right)\right]+\imath_{2 p} R_{3} \sin \left[\pi\left(\Phi_{3}+\mu_{2 p}+\lambda_{1}\right)\right]=0 .
\end{aligned}
$$

Nous renvoyons encore au $\S 10$ pour la discussion de l'éventualité où $\lambda_{1}-\lambda_{2}$ serait un entier.

On voit donc que l'ensemble des relations (13) et (14) constitue un système de quatre équations 
linéaires et homogènes en $R_{2} \cos \pi \Phi_{2}, \quad R_{2} \sin \pi \Phi_{2}, \quad R_{4} \cos \pi \Phi_{4}$ et $R_{4} \sin \pi \Phi_{4}$ qui admet une solution non nulle si et si seulement leur déterminant est nul. Cela donne l'équation de définition de $\lambda_{2}=\lambda_{\text {. }}$

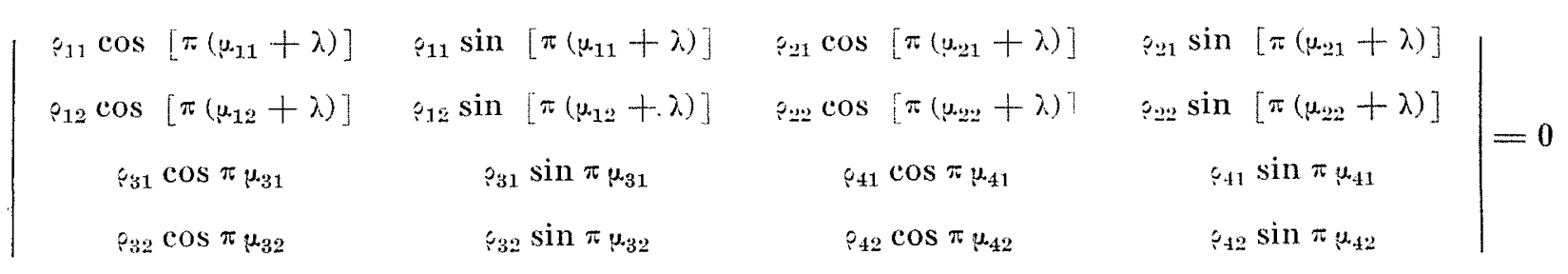

En opérant de même avec $\left(13^{\prime}\right)$ et $\left(14^{\prime}\right)$, on vérifie que $\lambda_{1}$ est encore solution de (15). C'est l'équation (E) aux exposants caractéristiques que nous nous proposions de former. On voit d'abord que si (15) admet une racine $\lambda$, tout nombre de la forme $\lambda+r, r$ étant un entier quelconque, sera encore racine de (15). Cela prouve, comme nous l'annoncions ci-dessus, que les exposants $\lambda_{1}$ et $\lambda_{2}$ ne peuvent être définis qu'à un entier additif près. Mais des considérations d'ordre géométrique et physique, dont on verra plus loin des exemples, permettent toujours de lever cette indétermination. Dans ce travail, nous supposerons connu à priori le voisinage du point $\Omega\left(z_{n, m}\right)$ appartenant à $\delta$, ce qui suffira pour fixer sans. ambiguïté les valeurs de $\lambda_{1}$ et de $\lambda_{2}$.

Observons que chaque élément des deux premières lignes de (15) est linéaire et homogène en $\cos \pi \lambda$ et $\sin \pi \lambda ;(15)$ est donc une équation du second degré $\operatorname{tg} \pi \lambda$ dont les racines sont nécessairement réelles d'après les résultats de [2] et de [5], rappelés au cours du paragraphe 7 . Nous pouvons donc dans tous les cas calculer élémentairement $\lambda_{1}$ et $\lambda_{2}$.

9. Ceci étant, l'ensemble des équations (13) et (14) - où i.z sera regardé comme connu -..nous donnera $R_{2} \cos \pi \Phi_{2}, R_{2} \sin \pi \Phi_{2}, R_{4} \cos \pi \Phi_{4}$ et $R_{4} \sin \pi \Phi_{4}$ à un facteur arbitraire près; dans les cas courants, en effet, la matrice (15) est de rang 3. Il s'ensuil que $\operatorname{tg} \pi \Phi_{2}$ et $\operatorname{tg} \pi \Phi_{4}$ sont connues sans ambiguïté, ce qui permet de déterminer $\Phi_{2}$ et $\Phi_{4}$ à un entier additif près. On précisera la détermination de ces paramètres d'après le signe de la constante multiplicative, introduite ci-dessus pour que $R_{2}$ et $R_{4}$ soient $>0$. Donc $R_{2}$ el $R_{4}$ seront déterminés à un facteur constant $>0$ près.

On opérera d'une manière toute semblable pour calculer $R_{1}, R_{3}$, $\Phi_{1}$ et $\Phi_{3}$ à partir de $\left(13^{\prime}\right)$ et $\left(14^{\prime}\right)$.

En résumé, la méthode permet de calculer facilement les exposants caractéristiques $\lambda_{1}$ et $\lambda_{2}$ et les rapports $K_{3} / K_{1}, K_{4} / K_{2}$. Il en résulte la connaissance des parties principales de $F(\zeta)$ et de $Z(\zeta)$, donc, par intégration, de celles de $f(\zeta)$ et $z(\zeta)$.

10. Si (15) admet une racine double en $\operatorname{tg} \pi \lambda$, ou, plus précisément, si $\lambda_{1}-\lambda_{2}$ est un entier, les raisonnements du paragraphe 8 doivent subir une légère retouche.

Supposons que $\log \zeta$ se réduise à sa détermination réelle pour $\zeta=\zeta>0$; alors (12) demeure valable, à condition de remplacer entre les accolades du second membre $\zeta^{\lambda_{1}-\lambda_{2}}$ par log $\zeta_{\text {. Les }}$ raisonnements du paragraphe 8 s'appliquent encore et prouvent que les équations (13) et (13') subsistent intégralement.

Faisons, maintenant, $\zeta=|\xi| e^{\pi i},(\xi<0)$, el portons dans (7) les valeurs $(9)$ et $\left(10^{\prime}\right)$ de $\mathrm{F}$ et de $Z$. Après la suppression du facteur réel $|\xi|^{\lambda}$, il y aura lieu de satisfaire deux conditions du type :

$$
\mathrm{I}_{m} e^{\lambda \pi i}\left[\alpha_{p} \mathrm{~K}_{1}+\beta_{p} \mathrm{~K}_{3}+\left(x_{p} \mathrm{~K}_{2}+\beta_{p} \mathrm{~K}_{4}\right) \pi i+\left(\alpha_{p} \mathrm{~K}_{2}+\beta_{p} \mathrm{~K}_{1}\right) \log |\xi|+\ldots \vdots=0,(p=1,2),\right.
$$

où le symbole... désigne encore une expression telle que :

$$
\xi \sum_{q=0}^{1+=\infty} a_{q} \xi^{q}+\xi \log |\xi| \sum_{q=0}^{t=-\infty} b_{q} \xi^{q}
$$

I)es raisonnements ćlémentaires montrent alors que l'on doit avoir :

$$
\mathrm{I}_{m}\left[e^{\lambda i}\left(\alpha_{p} \mathrm{~K}_{2}+\beta_{p} \mathrm{~K}_{4}\right)\right]=0, \quad(p=1,2),
$$

¿quation identique à (14) et la relation qui remplace (14') :

$$
\begin{gathered}
\imath_{1 p} \mathrm{R}_{1} \sin \left[\pi\left(\Phi_{1}+\mu_{1 p}+\lambda\right)\right]+\rho_{2 p} \mathrm{R}_{3} \sin \left[\pi\left(\Phi_{3}+\mu_{2 p}+\lambda\right)\right] \\
=-\pi\left\{\imath_{1 p} \mathrm{R}_{2} \cos \left[\pi\left(\Phi_{2}+\mu_{1 p}+\lambda\right)\right]+\imath_{2 p} \mathrm{R}_{4} \cos \left[\pi\left(\Phi_{1}+\mu_{2 p}+\lambda\right)\right]\right\} \quad(p=1.2) .
\end{gathered}
$$

On voit que la détermination des constantes $R_{2}, R_{4}, \Phi_{2}, \Phi_{4}$, se fera encore selon le schéma indiqué au paragraphe 9 . Par contre, le caleul de $R_{1}, R_{3}$, $\Phi_{1}$ et $\Phi_{3}$ exigera la discussion du système (13') et (16), non homogène (on supposera que le second membre de (16) a été déterminé comme il vient d'être dit) à déterminant nul. Nous donnerons dans [5] une démonstration indirecte de la compati- 
bilité de $\left(13^{\prime}\right)$ et de (16). Nous nous bornerons ici à faire remarquer que dans les applications techniques courantes, les coefficients de $\left(13^{\prime}\right)$ et de (16) ont une forme très simple et la résolution de ce système sera, pratiquement, immédiate.

11. Il serait aisé de développer le premier membre de (15) : il nous a semblé préférable de conserver la forme symétrique du déterminant, plus facile à se rappeler et dont le calcul est immédiat dans la plupart des applications. En utilisant les formules (11), on voit que (15) s'écrit :

$\begin{array}{cccc}\mathrm{Q}_{n 1} \cos \pi \lambda-\mathrm{P}_{n 1} \sin \pi \lambda & \mathrm{Q}_{n 1} \sin \pi \lambda+\mathrm{P}_{n 1} \cos \pi \lambda & \mathrm{N}_{n 1} \cos \pi \lambda-\mathrm{M}_{n 1} \sin \pi \lambda & \mathrm{N}_{n 1} \sin \pi \lambda+\mathrm{M}_{n 1} \cos \pi \lambda \\ \mathrm{Q}_{n 2} \cos \pi \lambda-\mathrm{P}_{n 2} \sin \pi \lambda & \mathrm{Q}_{n 2} \sin \pi \lambda+\mathrm{P}_{n 2} \cos \pi \lambda & \mathrm{N}_{n 2} \cos \pi \lambda-\mathrm{M}_{n 2} \sin \pi \lambda & \mathrm{N}_{n 2} \sin \pi \lambda+\mathrm{M}_{n 2} \cos \pi \lambda \\ \mathrm{Q}_{m 1} & \mathbf{P}_{m 1} & \mathbf{N}_{m 1} & \mathbf{M}_{m 1} \\ \mathrm{Q}_{m 2} & \mathbf{P}_{m 2} & \mathrm{~N}_{m 2} & \mathbf{M}_{m 2}\end{array} \mid \begin{gathered}(17) \\ =0\end{gathered}$

D'après cela et la formule (11), les équations (13) et (13'), (14) et (14') s'écrivent respectivement :

$$
\begin{aligned}
& \mathrm{K}_{2}^{\prime \prime} \mathrm{Q}_{m p}+\mathrm{K}_{2}^{\prime} \mathrm{P}_{m p}+\mathrm{K}^{\prime \prime}{ }_{4} \mathrm{~N}_{m p}+\mathrm{K}_{4}{ }_{4} \mathrm{M}_{m p}=0 ; \quad(p=1,2) ; \\
& \mathrm{K}^{\prime \prime}{ }_{1} \mathrm{Q}_{m p}+\mathrm{K}_{1}^{\prime} \mathbf{P}_{m p}+\mathrm{K}^{\prime \prime}{ }_{3} \mathrm{~N}_{m p}+\mathrm{K}_{3}{ }_{3} \mathrm{M}_{m p}=0 ; \quad(p=1,2) \text {; } \\
& \mathrm{K}^{\prime \prime}{ }_{2}\left(\mathrm{Q}_{n p} \cos \pi \lambda_{2}-\mathrm{P}_{n p} \sin \pi \lambda_{2}\right)+\mathrm{K}_{2}^{\prime}{ }_{2}\left(\mathrm{Q}_{n p} \sin \pi \lambda_{2}+\mathrm{P}_{n p} \cos \pi \lambda_{2}\right)+ \\
& +\mathrm{K}^{\prime \prime}{ }_{4}\left(\mathrm{~N}_{n p} \cos \pi \lambda_{2}-\mathrm{M}_{n p} \sin \pi \lambda_{2}\right)+\mathrm{K}_{4}^{\prime}\left(\mathrm{N}_{n p} \sin \pi \lambda_{2}+\mathrm{M}_{n \eta} \cos \pi \lambda_{2}\right)=0, \quad(p=1,2) ; \\
& \mathrm{K}^{\prime \prime}{ }_{1} \cdot\left(\mathrm{Q}_{n p} \cos \pi \lambda_{1}--\mathrm{P}_{n p} \sin \pi \lambda_{22}\right)+\mathrm{K}_{1}^{\prime}\left(\mathrm{Q}_{n p} \sin \pi \lambda_{1}+\mathrm{P}_{n p} \cos \pi \lambda_{1}+\right. \\
& +\mathrm{K}^{\prime \prime}{ }_{3}\left(\mathbf{N}_{n p} \cos \pi \lambda_{1}-\mathrm{M}_{n p} \sin \pi \lambda_{1}\right)+\mathrm{K}_{3}^{\prime}\left(\mathbf{N}_{n p} \sin \pi \lambda_{1}+\mathrm{M}_{n p} \cos \pi \lambda_{1}\right)=0, \quad(p=1,2) \text {. }
\end{aligned}
$$

Enfin, l'équation (16) se transforme en la suivante :

$$
\begin{array}{r}
\mathrm{K}_{{ }_{1}}{ }_{1}\left(\mathrm{Q}_{n p} \cos \pi \lambda-\mathrm{P}_{n p} \sin \pi \lambda\right)+\mathrm{K}_{1}^{\prime}\left(\mathrm{Q}_{n p} \sin \pi \lambda+\mathrm{P}_{n p} \cos \pi \lambda\right) \\
+\mathrm{K}_{3}{ }_{3}\left(\mathrm{~N}_{n p} \cos \pi \lambda-\mathrm{M}_{n p} \sin \pi \lambda\right)+\mathrm{K}_{3}^{\prime}\left(\mathrm{N}_{n p} \sin \pi \lambda+\mathrm{M}_{n p} \cos \pi \lambda\right) \\
=-\pi\left[\mathrm{K}_{2}^{\prime}\left(\mathrm{Q}_{n p} \cos \pi \lambda-\mathrm{P}_{n p} \sin \pi \lambda\right)-\mathrm{K}_{2}{ }_{2}\left(\mathrm{Q}_{n p} \sin \pi \lambda+\mathrm{P}_{n p} \cos \pi \lambda\right)\right. \\
\left.+\mathrm{K}_{4}^{\prime}\left(\mathrm{N}_{n p} \cos \pi \lambda--\mathrm{M}_{n p} \sin \pi \lambda\right)-\mathrm{K}^{\prime \prime}{ }_{4}\left(\mathrm{~N}_{n p} \sin \pi \lambda+\mathrm{M}_{n p} \cos \pi \lambda\right)\right]
\end{array}
$$

Ainsi, pratiquement, on n'aura pas à mettre les quantités complexes $\alpha_{p}, \beta_{p}, \mathrm{~A}_{p}, \mathrm{~B}_{p}(p=1,2)$ sous la forme trigonométrique (11); celle-ci n'est utile que pour effectuer commodément quelques calculs intermédiaires.

La marche des calculs précédents est présentée dans [2] d'une manière différente. PoloubariNova-Kotchina forme d'abord l'équation du second degré dont les racines sont $e^{2 \pi i \lambda_{1}}$ et $e^{2 \pi i \lambda_{2}}$; il nous semble que notre variante est plus directe et plus rapide. En second lieu, l'auteur précité n'aborde pas la détermination des constantes $K_{j}[\mathrm{Cf}$. (9) et (11)]. On verra au chapitre suivant l'intérêt pratique de ce complément de la théorie.

Cependant, redisons une fois encore que l'essentiel du chapitre II se trouvè déjà dans l'ouvrage fondamental $[2]$.

\section{CHAPITRE III}

\section{ETUDE DE QUELQUES CAS PARTICULIERS}

12. Nous allons illustrer les généralités du chapitre précédent en discutant d'une manière un peu détaillée les singularités de $f(z)$ aux sommets $z_{42}$ et $z_{34}$ de C. D'après les conventions adoptées aux paragraphes 2 et $3, z_{42}$ est commun à une équipotentielle $\mathrm{C}_{2}$ et à une ligne libre $\mathrm{C}_{4}$. Pratiquement, la question revient à étudier l'al- lure de $\mathrm{C}_{4}$ dans le voisinage d'une paroi perméable. De même, l'étude du voisinage de $z_{34}$ nous permettra de préciser l'allure d'une ligne libre dans le voisinage du point où elle se raccorde à une surface de suintement.

La plupart des résultats ci-après se trouvent déjà dans la thèsè de Boreli [4]. Toutefóis, 
l'étude du point $z_{42}$ est faite, dans un travail précité, par des moyens élémentaires décrits au paragraphe 3 ; la discussion qu'on va lire est, par ailleurs, plus complète.

13. Faisons $n=4, m=2$ dans les formules du paragraphe 6 . Il en résulte que pour $a \leqslant \xi \leqslant 0$, $\eta=0$, nous avons $[\mathrm{Cf}$. (3) et (4)] :

$$
\begin{aligned}
& \mathrm{I}_{m}(\mathrm{~F})=0 \\
& \mathrm{I}_{m}(i \mathrm{~F}+k \mathrm{Z})=0
\end{aligned}
$$

ce qui permet d'écrire :

$$
\left.\begin{array}{llll}
Q_{41}=1 ; & P_{41}=0 ; & N_{41}=0 ; & M_{41}=0 ; \\
Q_{42}=0 ; & P_{42}=1 & N_{42}=k ; & M_{42}=0 .
\end{array}\right\}
$$

Posons [Cf. (1)] :

$$
a_{2}=\sin \pi \alpha ; \quad b_{2}=-\cos \pi \alpha ;
$$

$\pi \approx$ est, d'après cela, l'angle d'inclinaison de la paroi perméable sur l'horizontale. Il s'ensuit (Cf. \$ 2) que les conditions frontières le long de $\mathrm{C}_{2}$ s'écrivent :

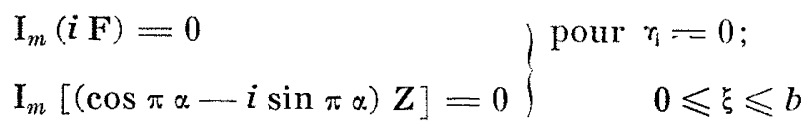

On en déduit (Cf. $\S 6$ ) :

$$
\left.\begin{array}{l}
\mathrm{Q}_{21}=0 ; \mathrm{P}_{21}=1 ; \mathrm{N}_{21}=0 ; \quad \mathrm{M}_{21}=0 ; \\
\mathrm{Q}_{22}=0 ; \mathrm{P}_{22}=0 ; \mathrm{N}_{22}=\cos \pi \alpha ; \mathrm{M}_{22}=-\sin \pi \alpha .
\end{array}\right\}
$$

14. Portons les valeurs (21) et (22) dans l'équation (17); il vient :

$\left|\begin{array}{cccc}\cos \pi \lambda & \sin \pi \lambda & 0 & 0 \\ -\sin \pi \lambda & \cos \pi \lambda & k \cos \pi \lambda & k \sin \pi \lambda \\ 0 & 1 & 0 & 0 \\ 0 & 0 & \cos \pi \alpha & -\sin \pi \alpha\end{array}\right|=0$

En développant le premier membre de (23) suivant les éléments de la troisième ligne, on trouve:

$$
k \cos \pi \lambda \sin [\pi(\lambda+\alpha)]=0
$$

en sorte que, $r$ et $r_{1}$ étant des entiers quelcon- ques (dont on précisera ultérieurement les valeurs):

$$
\lambda_{1}=(1 / 2)+r ; \quad \lambda_{2}=-\alpha+r_{1} .
$$

Notons que $\lambda_{1}-\lambda_{2}$ se réduit à un entier si $\alpha=1 / 2$; la paroi perméable est alors verticale. D'après ce qu'on a vu au paragraphe 7 , les singularités de $F$ et de $Z$ sont, pour $\alpha=1 / 2$, d'un type différent $\left[\mathrm{Cf}\right.$. (9) et $\left.\left(10^{\prime}\right)\right]$ : nous discuterons au paragraphe 18 ce cas particulier en détail. Jusqu'à nouvel ordre, nous supposons $\alpha \neq 1 / 2$.

15. Passons maintenant à la détermination des constantes $\mathrm{K}_{j}(j=1,2,3,4)$. Pour $\lambda=\lambda_{1}=1 / 2$, la matrice (23) devient :

$\begin{array}{cccc}0 & 1 & 0 & 0 \\ -1 & 0 & 0 & k \\ 0 & 1 & 0 & 0 \\ 0 & 0 & \cos \pi \alpha & -\sin \pi \alpha\end{array}$

Elle est de rang 3; si donc $R_{3}$ est une constante réelle, arbitraire - dont nous préciserons ultérieurement le signe _- on a :[Cf $\left(18^{\prime}\right)$ et $\left.\left(19^{\prime}\right)\right]$ :

$$
\mathrm{K}_{1}=i k \mathrm{R}_{3} \cos \pi \alpha ; \quad \mathrm{K}_{3}=\mathrm{R}_{3} e^{i \pi u} .
$$

Si, maintenant, on prend : $\lambda=\lambda_{2}=-\alpha$, la matrice (23) se réduit à :

$\begin{array}{cccc}\cos \pi \alpha & -\sin \pi \alpha & 0 & 0 \\ \sin \pi \alpha & \cos \pi \alpha & k \cos \pi \alpha & -k \sin \pi \alpha \\ 0 & 1 & 0 & 0 \\ 0 & 0 & \cos \pi \alpha & -\sin \pi \alpha\end{array}$

De (18) et (19), il résulte alors, $R_{4}$ étant une constante réelle arbitraire dont le signe est à préciser) :

$$
\mathrm{K}_{2}=0 ; \quad \mathrm{K}_{4}=\mathrm{R}_{4} e^{i \pi a} .
$$

Alors (9) et (10) entraînent :

$$
\mathrm{F}(\zeta)=i k \mathrm{R}_{3} \cos \pi \alpha \zeta^{(1 / 2)+r} u_{1}(\zeta)
$$$$
Z(\zeta)=e^{\pi i a}\left[\mathrm{R}_{3} \zeta^{(1 / 2)+r} u_{1}(\zeta)+\mathrm{R}_{4} \zeta^{-a+r_{1}} u_{2}(\zeta)\right]
$$

Admettons - ce qui ne restreint pas la généralité - que $z_{42}=0, f_{42}=f\left(z_{42}\right)=0$. On déduit de là :

$$
\begin{aligned}
& f(\zeta)=\frac{i k \mathrm{R}_{3} \cos \pi \alpha}{(1 / 2)+r+1} \zeta^{(1 / 2)+r+1} v_{1}(\zeta) \\
& \left.z(\zeta)=e^{\pi i a}\left[\frac{\mathrm{R}_{3}}{(1 / 2)+r+1} \zeta^{(1 / 2)+r+1} v_{1}(\zeta)+{\frac{\mathrm{R}_{4}}{r_{1}-\alpha+1}}^{\zeta-a+r_{1}+1} v_{2}(\zeta)\right]\right\}
\end{aligned}
$$

où $v_{1}(\zeta)$ et $v_{2}(\zeta)$ sont encore des fonctions holomorphes à l'origine, telle que $v_{1}(0)=v_{2}(0)=1$; comme $1>1-\alpha>0$, on voit déjà que $z(0)=0$ exige que : $r_{1} \geqslant 0, r \geqslant-1$. 
16. Nous sommes maintenant en mesure de préciser les valeurs des entiers $r$ et $r_{1}$ et les signes des paramètres $R_{3}$ et $R_{4}$.

Tout d'abord, observons que, d'après la définition même de $z_{42}$, la frontière $\Gamma$ est constituée, dans le voisinage du point $f_{42}$, par le segment $\Gamma_{2}$ d'équation $\varphi=0, \psi \leqslant 0$ et le segment $\Gamma_{4}$, d'équation $\psi=0, \varphi \geqslant 0$, le domaine $\Delta$ étant contenu dans le quadrant: $\varphi \geqslant 0, \psi \leqslant 0$. Il en résulte [Cf. $\left.\left(24^{\prime}\right)\right]$ qu'on a pour $x-(1 / 2)<0$ (done, pour $\cos \pi \alpha>0$ ) :

$$
\begin{array}{r}
{[p+(1 / 2) \pi=\arg /(\zeta)=-(\pi / 2)+2 q \pi,} \\
\text { pour } \zeta=\xi>0,
\end{array}
$$$$
(p+r+2) \pi=\arg f(\zeta)=2 q \pi,
$$

$$
\text { pour } \zeta=\zeta<0 \text {, }
$$

où $q$ est un entier quelconque et où $p$ est défini au moyen de $\mathrm{R}_{3}=\left|\mathrm{R}_{3}\right| e^{\text {rip }},(p=0,1)$. On voit donc que $p=1, r=-1$, en sorte que, $R_{3}$ étant pris positif, (24) s'écrit pour $x-(1 / 2)<0$ :

$$
\begin{gathered}
\mathrm{F}(\zeta)=-i k \cos \pi x \mathrm{R}_{3,} \zeta^{-1 / 2} u_{1}(\zeta) ; \\
Z(\zeta)=e^{\pi i a}\left[-\mathrm{R}_{: 3} \zeta^{-1 / 2} u_{1}(\zeta)+\mathrm{R}+\zeta-a+r_{1} u_{2}(\zeta)\right]
\end{gathered}
$$

Pour $\alpha-(1 / 2)>0$, on fera dans $(25): \mathrm{R}_{: 3}<0$; car alors $\cos \pi \alpha<0$.

Il est clair qu'aucune des constantes $R_{3}$ et $R_{4}$ ne peut être nulle. Si, par exemple, on avait $\mathrm{R}_{3}=0$, (25) donnerait : $\mathrm{F}(\zeta)=0$, ce qui est impossible. De même l'hypothèse $\mathrm{R}_{4}=0$ entraînerait :

$$
\Omega(\zeta)=\frac{F(\zeta)}{Z(\zeta)}=\frac{i k \cos \pi \%}{\rho^{i \pi t}}=C^{t+}
$$

Le champ des vitesses serail uniforme dans le voisinage de la singularité, done uniforme partoul, ce qui est absurde. Posons $c=-\left(\mathbf{R}_{4} / \mathbf{R}_{3}\right)$; appelons encore $w_{1}(\zeta)=u_{2} / u_{1}$; c'est une fonction holomorphe à l'origine telle que $w_{1}(0)=1$; (25) donne: :

$$
\frac{1}{\Omega(\zeta)}=\frac{e^{\pi i a}}{i k \cos \pi \%}\left[1+c \zeta_{1}+(1 / \mu)-n w_{1}(\zeta)\right)
$$

On en déduil aisément le développement limité valable dans le domaine de $\zeta=0$, chaque fois que : $r_{1}+(1 / 2)--x>0:$

$$
\Omega(\zeta)=i k \cos \pi x e^{-i \pi a}\left\lceil1 \ldots \left(\zeta^{n_{1}+(1 / 2)-a} w_{1}(\zeta)+\ldots\right.\right.
$$

où le symbole... désigne une série entière en $c \zeta^{r_{1}+(1 / 2)-a} w_{1}(\zeta)$.

D'après ce que nous avons vu tout à l'heure : $r_{1} \geqslant 0$; la formule (26') s'applique chaque fois que $1 / 2-\alpha>0$.

Pour préciser le choix de $r_{1}$, des considérations d'ordre géométrique et physique deviennent nécessaires. Nous avons admis que $\gamma$ était un contour continu. Cette hypothèse exprime une propriété commune à tous les écoulements physiquement acceptables : le module du vecteur vitesse $\vec{V}$ doit avoir une valeur bien déterminée en chaque point de D - valeur qui peut, évenluellement, devenir infinie. Il en résulte que les coordonnées $u, v_{1}$ du point :

$$
\Omega_{42}=\Omega\left(z_{42}\right)=u-i v=u+i v_{1}
$$

s'obtiennent en résolvant le système :

$$
\begin{gathered}
u^{2}+v^{2}-k v=0 \\
u \cos \pi x-v \sin \pi x=0
\end{gathered}
$$

dont les racines sont:

$$
\begin{array}{ll}
u=0 & u=k \sin \pi x \cos \pi \alpha ; \\
v_{1}=0 & v_{1}=k \cos ^{2} \pi . .
\end{array}
$$

Or, compte tenu du sens général du mouvement - qui est le sens positif de $\mathrm{O} x$ - on roit que $u \geqslant 0$ sur $\gamma_{2}$; on en déduit (Cf. 27) que $v_{1}$ et $(1 / 2)-x$ sont du même signe le long de cette ligne. Cela montre que $\Omega(0)$ est défini par :

$$
\Omega(0)=\Omega_{42}=0,
$$

si $\% \cdot(1 / 2)>0$

et :

$$
\Omega(0)=\Omega_{4: 2}=i k \cos \pi \propto c^{-i \pi r},
$$

si $z-(1 / 2)<0$; ces conclusions sont en accord avec $(26)$ et $\left(26^{\prime}\right)$, où $r_{1} \geqslant 0$. Enfin, $\left(27_{1}\right)$ montre immédiatement que $\gamma_{4}$ est située dans le domainc $v \geqslant 0$, quel que soit $\alpha, 0<\alpha<1$. Rappelons aussi que si $\zeta=\xi$ décrit l'axe réel dans le sens positif, $\mathcal{Q}(\zeta)$ décrit $\gamma$ dans le sens direct, parcourant, dans cet ordre, $\gamma_{4}$ et $\gamma_{2}$.

Deux cas sont, dès lors, à considérer :

$1^{\circ} \alpha-(1 / 2)>0$. Alors $\gamma$ contient le point $\Omega(0)=\Omega_{42}=0$. Dans le voisinage de $\Omega_{42}$, le domaine ò est défini par les inégalités :

$$
\begin{gathered}
u^{2}+v^{2}-k v \geqslant 0 ; \\
u \cos \pi x \cdots-v \sin \pi x \leqslant 0,
\end{gathered}
$$

et arg $\Omega(\xi)$ passe de 0 à $\pi[(1 / 2)-\alpha]$ lorsque le point $\zeta=\xi$ traverse l'origine en décrivant $\eta_{1}=0$ dans le sens positif. 
Contròlons ces prévisions en discutant (26). Il est clair que $\Omega(0)=0$ entraine :

$$
r_{1}+(1 / 2)-\alpha<0 .
$$

Comme $r_{1} \geqslant 0$ et $|(1 / 2)-\alpha|<1$, cela exige $r_{1}=0$. Alors on déduit de (26) le développement limité :

$$
\underline{\Omega}(\zeta)=\frac{k \cos \pi x e_{(1 / 2)-a] \pi i}}{c} \zeta^{(1-(1 / 2)}\left[1-(1 / c) \zeta^{(1-(1 / 2)}+\mathrm{O}\left(\zeta^{2}(a-1)\right],\right.
$$

où le symbole $O(\zeta)$ désigne, suivant l'usage, un infiniment petit d'ordre $\zeta$. On tire de là les équations paramétriques de $\gamma$ dans le voisinage de $\Omega=0$.

$$
\begin{aligned}
& \left.u(\xi)=\frac{k \cos \pi \alpha}{c}\left|\xi_{i}^{a-1 / 2}-\frac{k \cos \pi \alpha \sin \pi x}{c^{2}}\right| \xi^{2} a-1+O\left(\zeta^{3 a-\cdots ; / 2}\right) ;\right) \\
& D(\xi)=\frac{k \cos ^{2} \pi \alpha}{c^{2}}|\xi|^{2 a-1}+O\left(\xi^{3 a-1 ; / 2)}\right) \\
& \text { pour } \because-1: \quad \zeta=\ddot{\zeta}<0 \text {. }
\end{aligned}
$$

el :

$$
\left.\begin{array}{l}
u(\xi)=\frac{k \cos \pi x \sin \pi \alpha}{c}|\xi|^{a-1 / 2}\left[1-\frac{1}{c} \xi^{a-1 / 2}+\mathrm{O}\left(\xi^{2 a-1}\right)\right] \\
\left.v(\xi)=\frac{k \cos ^{2} \pi \alpha}{c}|\xi|^{a-1 / 2}\left[1-\frac{1}{c} \xi^{a-1 / 2}+\mathrm{O}\left(\zeta^{2 a-1}\right)\right]\right) ;
\end{array}\right\} \text { pour } \gamma: \quad \zeta=\xi>0 .
$$

Comme $\cos \pi \alpha<0$, on voit que $u$ ne peut être positif sur $\gamma_{4}$ et $v_{1}$ n'est négatif sur $\gamma_{2}$, dans le voisinage de l'origine $\zeta=0$, que si :

$$
c=-\left(\mathbf{R}_{4} / \mathbf{R}_{3}\right)<0 \text {. }
$$

Ainsi donc, la condition $\Omega(0)=0$ suffit pour déterminer la valeur de $r_{1}$; par contre, la discussion des signes de $R_{4}$ exige la connaissance, à priori, de la forme qu'affecte le domaine o dans roisinage de l'origine $\zeta=0$, que si :

$2^{\circ} \alpha-(1 / 2)<0$. Alors $\gamma$ contient le point $Q(0)=\Omega_{42}=k \cos \pi x e^{[(1 / 2)-\alpha] \pi i}$.

Supposons que le voisinage de $\Omega_{4: 2}$ du domaine o soit, dans ce cas, défini par l'un des deux groupes d'inégalités :

$$
\begin{aligned}
& \text { (I) } \mid n^{2}+v^{2}-k \cdot v \geqslant 0 \text {; } \\
& 1 u \cos \pi x-v \sin \pi \alpha \leqslant 0 \text {. } \\
& \text { (II) }\left\{\begin{array}{l}
u^{2}+v^{2}-k v \leqslant 0 ; \\
u \cos \pi x-v \sin \pi x \geqslant 0 .
\end{array}\right.
\end{aligned}
$$

Dans le cas $(\boldsymbol{I}), \arg \left[\Omega(\zeta)-\Omega_{42}\right]$ passe de $\pi(1-2 \alpha)$ à $\pi[(1 / 2)-\alpha]$ lorsque $\xi$ traverse l'origine par valeurs croissantes; dans le cas (II), $\arg \left[\Omega(\zeta)-\Omega_{42}\right]$ passe, dans les mêmes conditions, de $-2 \pi_{\alpha} \dot{\mathrm{a}}-\pi[\alpha+(1 / 2)]$.

Comparons ces résultats, à priori, avec $\left(26^{\prime}\right)$. Posons $c=|c| e^{p i \pi}, p=0,-1$. Il vient :

$$
\arg \left[\Omega(\xi)--\Omega_{42}\right]=\pi[(3 / 2)-x+p]+2 \pi q,
$$

$$
\text { pour } \zeta=\xi>0 \text {; }
$$

$$
\begin{array}{r}
\arg \left[\Omega(\xi)-Q_{42}\right]=\pi\left[2-2 \%+p+r_{1}\right]+2 \pi q, \\
\text { pour } \zeta=\xi<0,
\end{array}
$$

$q$ étant un entier quelconque. On voil alors immédiatement que $p=-1, r_{1}=0$ dans le cas (I) et que $p=0, r_{1}=0$ dans le cas (II). Dans le cas (I), on prendra pour $R_{4}$ un nombre positif et un nombre négatif dans le cas II.

En résumé, quel que soil le signe de $x-(1 / 2)$, on a: $\lambda_{1}=-1 / 2, \lambda_{2}=-\alpha$. Si $\alpha-(1 / 2)<0$, $\mathrm{R}_{3}>0$ [Cf. (25)]; de plus, $\mathrm{R}_{4}>0$ dans le cas (I) et $\mathrm{R}_{4}<0$ dans le cas (II). Si $\alpha-(1 / 2)>0$, $\mathrm{R}_{3}<0$ et $\mathrm{R}_{4}<0$ dans (25); dans (24), on lerail : $\mathrm{R}_{3}>0$ et $\mathrm{R}_{4}<0$.

17. Nous sommes maintenant en mesure d'étudier l'allure de la ligne $\mathrm{C}_{4}$ dans le voisinage de $z_{42}=0$. Dans le cas où $\alpha-(1 / 2)>0$, nous avons, en remplaçant $R_{3}$ par $-R_{3}{ }^{2}$ et $R_{4}$ par - $\mathrm{R}_{4}{ }^{2}$ dans les formules (25) (à noter qu'on a changé $R_{3}$ en $-R_{3}$ en passant de (24) à (25) :

$$
\begin{gathered}
f(\zeta)=2 i k \mathrm{R}_{3}^{2} \cos \pi g \zeta^{1 / 2} v_{1}(\zeta) \\
\left.z(\zeta)=e^{\pi i \alpha} \mid 2 \mathrm{R}_{3}^{2} \zeta^{1 / 2} \nu_{1}(\zeta) \cdots \frac{\mathrm{R}_{4}^{2}}{1-\alpha} \zeta^{1-a} \nu_{2}(\zeta)\right\rceil
\end{gathered}
$$

Ces formules conviennent aussi au cas (I), si $x-(1 / 2)<0$, à condition d'y remplacer $R_{i}{ }^{2}$ et $\mathrm{R}_{4}{ }^{2}$ par $-\mathrm{R}_{3}{ }^{2}$ et $-\mathrm{R}_{4}{ }^{2}$ respectivement.

Dans le cas (II) $[\alpha-(1 / 2)<0]$, il suffit d'y changer $R_{3}{ }^{2}$ en $-R_{2}{ }^{2}$. Plaçons-nous, pour fixer les idées, dans l'hypothèse où $x-(1 / 2)<0$ (cas I). D'après (28), nous pouvons écrire les dé- 
veloppements limités des équations paramétriques $x=x(\xi), y=y(\xi), \xi<0$ de $\mathrm{C}_{4}$ sous la forme valable pour de petites valeurs de $|\xi|$ :

$x(\xi)=2 \sin \pi \alpha \mathrm{R}_{3}^{2} \sqrt{|\xi|}-\frac{\mathrm{R}_{4}^{2}}{1-\alpha}|\xi|^{1-\alpha}+\mathrm{O}\left(|\xi|^{3 / 2}\right)$.

$$
y(\xi)=-2 \cos \pi \alpha \mathbf{R}_{3}{ }^{2} \sqrt{|\xi|}+\mathrm{O}\left(|\xi|^{3 / 2}\right)
$$

Ici, le terme $|\xi|^{1-\alpha}$ est infiniment petit par rapport à $|\xi|^{1 / 2}$. Il suffira d'y remplacer $R_{4}^{2}$ en $--R_{4}^{2}$ pour avoir les équations approchées de $\mathrm{C}_{4}$ dans le cas (II). A noter que ces deux éventualités (I) et (II) peuvent effectivement se produire; le sens de la courbure de $\mathrm{C}_{4}$ dans le voisinage de $z_{42}$ change d'un cas à l'autre. Nous laissons au lecteur le soin de vérifier ce point; on trouvera dans la thèse de Boreur [4] tous les détails désirables.

Enfin, les formules (29) conviennent encore au cas $\alpha-(1 / 2)>0$, à condition d'y remplacer $R_{4}{ }^{2}$ par $-\mathbf{R}_{4}{ }^{2}$; mais alors dans $\left(29_{1}\right)$ le terme $|\xi|^{1 / 2}$ est un infiniment petit par rapport à $|\xi|^{1-a}$; de plus, $O\left(|\xi|^{3 / 2}\right)$ est à remplacer par $O\left(|\xi|^{2-a}\right)$. Sans entrer dans les détails, tirons de (29) quelques conséquences immédiates. D'abord, si $\alpha-(1 / 2)<0$, on voit que la pente de la tangente à $\mathrm{C}_{4}$ au point $z_{42}=0$ vaut - $\operatorname{cotg} \pi \alpha$; la ligne libre $\mathrm{C}_{4}$ est normale en $z_{42}$ à la paroi perméable $\mathrm{C}_{22}$. Au contraire, si $\alpha-(1 / 2)>0, \mathrm{C}_{4}$ admet en $z_{42}$ une tangente horizontale.

Etudions la courbure de $\mathrm{C}_{4}$ au point $z_{42}$. Si $\alpha-(1 / 2)<0$ (cas I), on tire de (29) l'équation cartésienne de $\mathrm{C}_{4}$, sous forme de développement limité, valable dans le voisinage de l'origine:

$$
x=-y \operatorname{tg} \pi \alpha-\frac{\mathrm{R}_{4}{ }^{2}}{\left(4 \mathrm{R}_{3}{ }^{4} \cos ^{2} \pi \alpha\right)^{1-\alpha}(1-\alpha)}(-y)^{2(1-\alpha)},
$$

Il suffirait de changer $\mathrm{R}_{4}{ }^{2}$ en $-\mathrm{R}_{4}{ }^{2}$ pour avoir ce développement dans le cas II. Il est aisé d'en déduire que la courbure de $\mathrm{C}_{4}$ en $z_{42}$ est infinie. On obtiendrait une conclusion identique en opérant directement sur les équations paramétriques (29) et en utilisant la relation classique :

$$
\mathfrak{R}=\frac{\left(x^{\prime 2}+y^{\prime 2}\right)^{3 / 2}}{x^{\prime} y^{\prime \prime}-y^{\prime} x^{\prime \prime}}
$$

où $\mathcal{R}$ désigne le rayon de courbure de $\mathrm{C}_{4}$ et où les accents notent la dérivation par rapport à la variable indépendante $t=|\xi|^{1 / 2}$.

La formule précédente permet aussi d'affirmer que $\mathrm{C}_{4}$ présente encore en $z_{42}$ une courbure infinie pour $\alpha-(1 / 2)>0$.

18. Il nous reste, maintenant, à étudier le cas singulier $\alpha=1 / 2$. Ici, l'équation caractéristique (23) se réduit à :

$$
\text { lk } \cos ^{2} \pi \lambda=0 \text {. }
$$

qui admet la racine double $\lambda=-1 / 2$, à un entier additif près. Les raisonnements des paragraphes 10 et 14 montrent que pour $\alpha=1 / 2$, on $a$ :

$$
\mathrm{K}_{2}=0 ; \quad \mathrm{K}_{4}=\mathrm{R}_{4} i,
$$

où $\mathbf{R}_{4}$ est une constante réelle arbitraire, dont le signe reste à préciser. Compte tenu de ces résultats, des formules (21) et (22), où l'on fera $\alpha=1 / 2$, les équations $\left(18^{\prime}\right)$ et $(20)$ s'écrivent :

$$
\mathrm{K}_{1}^{\prime}=0 ; \quad \mathrm{K}_{3}^{\prime}=0 ; \quad \mathrm{K}^{\prime \prime}{ }_{1}=-\pi k \mathrm{~K}^{\prime \prime}{ }_{4}
$$

On en déduit :

$$
\mathrm{K}_{1}=-i \pi k \mathrm{R}_{4} \quad \mathrm{~K}_{3}=\mathrm{R}_{3} i .
$$

Moyennant (9) et $\left(10^{\prime}\right)$, on peut donc écrire les expressions valables pour $|\zeta|$ petit :

$$
\begin{gathered}
\mathrm{F}(\zeta)=-i \pi k \mathrm{R}_{4} \zeta^{-(1 / 2)+r} u_{1}(\zeta) \\
Z(\zeta)=i \mathrm{R}_{3} \zeta^{-(1 / 2)+r} u_{1}(\zeta)+\mathrm{R}_{4} i \zeta^{(1 / 2)+r} \log \zeta u_{2}(\zeta)
\end{gathered}
$$

où $r$ est un entier que nous allons déterminer. A cet effet, il suffit de reprendre l'examen du plan $f$; les raisonnements du paragraphe 16 s'appliquent ici immédiatement et prouvent que $r=0$. On voit, du même coup, que $R_{4}>0$; en changeant $R_{4}$ en $R_{4}{ }^{2}$, nous avons les formules :

$$
\begin{gathered}
f(\zeta)=-2 i \pi k \mathrm{R}_{4}^{2} \zeta^{1 / 2} v_{1}(\zeta), \\
z(\zeta)=2 \mathrm{R}_{3} i \zeta^{1 / 2} v_{1}(\zeta) \\
+2 \mathrm{R}_{4}^{2} i \zeta^{1 / 2}\left[\log \zeta v_{2}(\zeta)-2 v_{3}(\zeta)\right]
\end{gathered}
$$

qui supposent encore : $z_{42}=0, f_{42}=0$ et où $v_{1}(\zeta), v_{2}(\zeta), v_{3}(\zeta)$ sont toujours des fonctions holomorphes à l'origine, telles que $v_{1}(0)=v_{2}(0)$ $=v_{3}(0)=1$. On déduit de là les équations paramétriques de $\mathrm{C}_{4}$ dans le voisinage de l'origine :

$$
\begin{aligned}
& \left.\begin{array}{r}
x(\xi)=-\mathrm{R}_{3} \vee T \xi\left|-2 \mathrm{R}_{4}^{2} \vee T \xi\right|(\log |\xi|-2) \\
+O\left(|\xi|^{3 / 2} \log |\xi|\right) ; \\
y(\xi)=-2 \mathrm{R}_{4}^{2} \pi \sqrt{|\xi|}+\mathrm{O}\left(|\xi|^{3 / 2} \log |\xi|\right) .
\end{array}\right\} \\
& \text { Il s'ensuit que : }
\end{aligned}
$$

$2^{\circ}$ La courbure de $\mathrm{C}_{4}$ en ce point est infinie.

19. Passons maintenant à l'étude du voisinage du sommet $z_{34}$, commun à une ligne de suintement $\mathrm{C}_{3}$ et à une ligne libre $\mathrm{C}_{4}$. La discussion étant analogue à celle qu'on vient de faire, nous nous bornerons à l'essentiel. Admettons, pour fixer les idées, que l'on rencontre les éléments contigus $\mathrm{C}_{3}, \mathrm{C}_{4}$ dans cet ordre et quand on décrit $\mathrm{C}$ dans le sens direct. Alors, nous ferons $n=3$ et $m=4$ dans les formules du paragraphe 6 . Donc, pour $\eta=0$ et $a \leqslant \xi \leqslant 0$, nous avons (Cf. le paragraphe 2) :

$$
\begin{aligned}
& \mathrm{I}_{m}\left(\mathrm{Z} e^{-i \pi \alpha}\right)=0 \\
& \mathrm{I}_{m}(\boldsymbol{i} \mathrm{F}+\boldsymbol{k} \mathrm{Z})=0
\end{aligned}
$$


en appelant $\pi \alpha$ l'angle d'inclinaison de la droite de suintement sur l'horizontale. Il s'ensuit [Cf. (11)] :

$\mathrm{Q}_{31}=0 ; \mathrm{P}_{31}=0 ; \mathrm{N}_{31}=\cos \pi \alpha ; \mathrm{M}_{31}=-\sin \pi \alpha ;$

$\mathrm{Q}_{32}=0 ; \mathrm{P}_{32}=1 ; \mathrm{N}_{32}=k \quad ; \mathrm{M}_{32}=0$

De même (Cf. le paragraphe 13), on a, pour $\eta=0$ et $O \leqslant \xi \leqslant b$ :

$$
\begin{aligned}
& \mathrm{I}_{m}(\mathrm{~F})=0 \\
& \mathrm{I}_{m}(i \mathrm{~F}+k \mathrm{Z})=0
\end{aligned}
$$

en sorte que :

$$
\begin{aligned}
& Q_{41}=1 ; \quad P_{41}=0 ; \quad N_{41}=0 ; \quad M_{41}=0 ; \\
& Q_{42}=0 ; \quad P_{42}=1 ; \quad N_{42}=k ; \quad M_{42}=0 .
\end{aligned}
$$

Les valeurs (30) et (31) des P, Q, M, N portées dans (17) donnent l'équation en $\lambda$ :

\begin{tabular}{cccc|}
0 & 0 & $\cos [\pi(\lambda \ldots \alpha)]$ & $\sin \lfloor\pi(\lambda \ldots \alpha)]$ \\
$\sin \pi \lambda$ & $\cos \pi \lambda$ & $k \cos \pi \lambda$ & $k \sin \pi \lambda$ \\
1 & 0 & 0 & 0 \\
0 & 1 & $k$ & 0
\end{tabular} \mid$=0$

qui, développée par rapport aux éléments de la troisième ligne du déterminant, s'écrit :

$$
k \cos [\pi(x-\lambda)] \sin \pi \lambda=0
$$

Les exposants caractéristiques sont donc :

$$
\lambda_{1}=r ; \quad \lambda_{2}=\alpha-(1 / 2)+r_{1}
$$

où $r$ et $r_{1}$ sont des entiers quelconques. On voit que $\lambda_{1}-\lambda_{2}$ ne sera un entier que si $\alpha=1 / 2$; la paroi de suintement est alors verticale et ce cas devra être examiné à part. Jusqu'à nouvel ordre, nous admettrons essentiellement : $\alpha \neq 1 / 2$.

20. Déterminons les constantes $K_{j}(j=1,2$, $3,4)$. Faisons $\lambda=\lambda_{1}=0$ dans la matrice du déterminant $(32)$; il vient :

$\begin{array}{cccc}0 & 0 & \cos \pi \alpha & \sin \pi \alpha \\ 0 & 1 & k & 0 \\ 1 & 0 & 0 & 0 \\ 0 & 1 & k & 0\end{array}$

Les formules $\left(\mathbf{1 8}^{\prime}\right)$ et $\left(\mathbf{1 9}^{\prime}\right)$ permettent aussitôt d'écrire :

$$
\mathrm{K}_{1}=-k \mathrm{R}_{3} \sin \pi \alpha ; \quad \mathrm{K}_{3}=\mathrm{R}_{3} e^{\pi t \alpha}
$$

De même, en faisant dans (32) :

$$
\lambda=\lambda_{2}=\alpha-1 / 2,
$$

on obtient le tableau :

$\begin{array}{cccc}0 & 0 & 0 & -1 \\ \cos \pi \alpha & \sin \pi \alpha & k \sin \pi \alpha & -k \cos \pi \alpha \\ 1 & 0 & 0 & 0 \\ 0 & 1 & k & 0\end{array}$

Les formules (18) et (19) donnent alors :

$$
\mathrm{K}_{2}=-k \mathrm{R}_{4} ; \quad \mathrm{K}_{4}=\mathrm{R}_{4} i
$$

Rappelons, une fois encore, que $R_{3}$ et $R_{4}$ sont des constantes réelles arbitraires dont le signe est à déterminer. Nous pouvons écrire [Cf. (9) et (10)] :

$$
\begin{aligned}
& \mathrm{F}(\zeta)=-k\left[\mathrm{R}_{3} \sin \pi \alpha \zeta^{r} u_{1}(\zeta)\right. \\
& \left.\quad+\mathrm{R}_{4} \zeta^{\alpha-(1 / 2)+r_{1}} u_{2}(\zeta)\right]
\end{aligned}
$$

$Z(\zeta)=\mathbf{R}_{3} e^{\pi i \alpha} \zeta^{r} u_{1}(\zeta)+\mathbf{R}_{4} i \zeta^{\alpha-(1 / 2)+r_{1}} u_{2}(\zeta)$,

où $u_{1}(\zeta), u_{2}(\zeta)$ sont, comme toujours, des fonctions holomorphes à l'origine, telle que $u_{1}(0)$ $=u_{2}(0)=1$. Il en résulte, en notant $z_{0}, f_{0}$ des constantes complexes arbitraires et $v_{1}(\zeta), v_{2}(\zeta)$ des fonctions holomorphes à l'origine, telles que $v_{1}(0)=v_{2}(0)=1$ :

$$
\begin{aligned}
f(\zeta)=f_{0} & -k\left[\frac{\mathrm{R}_{3} \sin \pi \%}{r+1} \zeta^{r+1} v_{1}(\zeta)\right. \\
& \left.+\frac{\mathrm{R}_{4}}{\alpha+(1 / 2)+r_{1}} \zeta^{\left(\alpha+(1 / 2)+r_{1}\right.} v_{2}(\zeta)\right] \\
z(\zeta)=z_{0} & +\frac{\mathrm{R}_{3}}{r+1} e^{\pi i \alpha} \zeta^{r+1} v_{1}(\zeta) \\
& +\frac{\mathrm{R}_{4} i}{\tau+(1 / 2)+r_{1}} \zeta^{\alpha+(1 / 2)+r_{1}} v_{2}(\zeta)
\end{aligned}
$$

Il faut ohserver que ces formules sont valables tant que $r \neq-1$. Sinon le développement de $z(\zeta)$ commencerait par un terme logarithmique. Alors $z(0)=\infty$, cas que nous écartons puisque $\mathrm{D}$ est borné. A noter encore que $\alpha+(1 / 2)+r_{1}$ ne peut être nul, car $r_{1}$ est entier et $0<\alpha<1$, $\alpha \neq 1 / 2$.

21. Il nous reste maintenant à déterminer les entiers $r$ et $r_{1}$; nous laissons au lecteur le soin de préciser les signes des constantes $R_{3}$ et $R_{4}$. En premier lieu, $f_{34}$ et $z_{34}$ sont bornés en module, par hypothèse. Les formules (36) montrent immédiatement que cela exige :

$$
r+1>0 ; \quad \alpha+(1 / 2)+r_{1}>0
$$


La premiere de ces relations peut encore s'écrire $r \geqslant 0$, car $r \neq \neq-1$.

Précisons la forme du domaine o dans le voisinage du point $\Omega_{34}$. D'après (5) et (6), ce point est à l'intersection des courbes :

$$
\begin{aligned}
& u^{2}+v^{2}-k v=0, \\
& u \cos \pi x-(v-k) \sin \pi x=0,
\end{aligned}
$$

c'est-à-dire l'un des points de coordonnées $u, v_{1}$ :

$$
\begin{aligned}
& u=0 ; \quad \nu_{1}=k ; \\
& u=-k \sin \pi x \cos \pi \alpha ; \quad v_{1}=k \sin ^{2} \pi x
\end{aligned}
$$

Le point $\Omega_{3 \pm}$ peut effectivement se confondre avec chacun des deux points ci-dessus. Cherchons par exemple une condition pour qu'on ait :

$$
\Omega_{34}=-k \sin \pi \alpha e^{-\pi i \alpha} .
$$

Supposons que dans le voisinage de $\Omega_{34}$ le domaine $\delta$ soit défini par les inégalités.

$$
\begin{aligned}
& u^{2}+v^{2}-k v \geqslant \leqslant 0 \\
& u \cdot \cos \pi \alpha-(v-k) \sin \pi \alpha \geqslant \leqslant 0,
\end{aligned}
$$

les signes $\geqslant$ et $\leqslant$ se correspondent dans les deux lignes

en sorte que le contour $\gamma$ présente en $\Omega_{34}$ un point anguleux. Il en résulte qu'aucune des constantes $R_{3}$ et $R_{4}$ ne sauraient être nulle, sinon, d'après (35), la correspondance :

$$
\Omega(\zeta)=[F(\zeta) / Z(\zeta)]
$$

serait holomorphe à l'origine et, en particulier, la langente à $\gamma$ ne subirait pas de discontinuité au point $\Omega_{34}$; d'ailleurs, $\Omega(\zeta)$ se réduirait à une constante.

Ceci étant, on tire de (35), en prenant $c=\left(\mathrm{R}_{4} / \mathrm{R}_{3}\right)$ ( $c$ étant une constante réelle finie, non nulle); $m=\alpha-(1 / 2)+r_{1}-r$ :

$$
\Omega(\zeta)=\frac{-k \sin \pi \alpha\left[1+(c / \sin \pi x) \zeta^{m} u(\zeta)\right.}{e^{\pi i \alpha}\left[1+c i \zeta^{m} w(\zeta) e^{-\pi i a}\right]}
$$

où $w(\zeta)$ est holomorphe à l'origine, telle que $w(o)=1$. Si $m<0$, on voit que $\Omega(0)=\Omega_{34}=i k$, solution écartée. Il faut donc supposer $m>0$ el on a bien alors :

$$
\Omega_{34}=-k \sin \pi \alpha e^{-\pi i \alpha}
$$

On peut, dès lors, écrire le développement limité suivant, valable dans le domaine de l'origine :

$$
\begin{aligned}
& \Omega(\zeta)-\Omega_{34} \\
& =-c k \sin \pi x e^{-\pi i \pi}\left[\frac{1}{\sin \pi x}+e^{-i \pi[a+(1 / 2)]}\right] \zeta^{m} \\
& \quad+O\left(\zeta^{m+1}\right)=-c k \cos \pi \alpha e^{-2 \pi i a} \zeta^{m}+O\left(\zeta^{m+1}\right) .
\end{aligned}
$$

Posons :

$$
c=|c| e^{\pi i p},(p=0,1) ; \cos \pi \alpha=|\cos \pi \alpha| e^{p^{\prime} i \pi},
$$

$p^{\prime}$ étant nul si $x<1 / 2$ et $p^{\prime}$ valant 1 si $\%>1 / 2$.

On voit donc que :

$$
\arg \left[\Omega(\xi)-\Omega_{34}\right]=\pi\left(p+p^{\prime}+1-2 \alpha\right)
$$

pour $\zeta=\xi>0$; et :

$\arg \left[\Omega(\xi)-\Omega_{34}\right]=\pi\left[p+p^{\prime}+(1 / 2)-\alpha+r_{1} \cdots r_{j}\right]$

pour $\zeta=|\xi| e^{x i}$. Or, l'examen du plan de la varialble permet aussitòt d'écrire, pour $x-(1 / 2)>0$ [Cf. $\left.\left(37^{\prime}\right)\right]$ :

$$
\arg \left[\Omega(\xi)-\Omega_{34}\right]=\pi(1-2 \alpha)
$$

pour $\zeta=\xi>0$ et :

$$
\arg \left[\Omega(\xi)-\Omega_{34}\right]=\pi[(1 / 2)-\alpha]
$$

pour $\zeta=|\xi| e^{\pi i}$, les arguments n'étant définis cu'à une même valeur additive près, valant 0 ou $\pi$ suivant le choix d'un voisinage particulier de ô près de $\Omega_{34}$. En égalant les valeurs correspondantes de l'argument, on trouve immcidiatement les relations où $p^{\prime}=1$ :

$$
p+p^{\prime}=0 \text { ou } 1 ; \quad r=r_{1} \text {. }
$$

I)'un autre côté, d'après (37): $r \geqslant 0$; donc $r_{1} \geqslant 0$ (Cf. 38). Or, si $r \geqslant 1$ (donc $r_{1} \geqslant 1$ ), le vecteur $z-z_{0}$ tourne d'un angle supérieur à $\pi$ quand le point $\zeta=\xi$ traverse l'origine $\zeta=0$ en décrivant l'axe réel [Cf. (36)]. Cette circonstance est inacceptable au point de vue physique. La validité de la solution construite exige, en effet, que le domaine $D$ soit tout entier situé d'un côté de la droite support du segment de suintement. Ainsi, nous sommes conduits à prendre $r=r_{1}$ $=0$; et les formules (36) s'écrivent, en définitive :

$$
\left.\begin{array}{rl}
f(\zeta)=f_{0}-k\left[\mathrm{R}_{3} \sin \pi \alpha \zeta v_{1}(\zeta)\right. \\
\left.+\left(2 \mathrm{R}_{4} / 2 \alpha+1\right) \zeta^{a+(1 / 2)} v_{2}(\zeta)\right] ; \\
z(\zeta)=z_{0}+\mathrm{R}_{3} e^{\pi i a \zeta v_{1}(\zeta)} \\
+i\left(2 \mathrm{R}_{4} / 2 \alpha+1\right) \zeta^{a+(1 / 2)} v_{2}(\zeta)
\end{array}\right\}
$$

La relation $r_{1}=r$, établie ci-dessus, entraîne : $m=\alpha-(1 / 2)$; or, les raisonnements précédents supposent justement que $m>0$, c'est-à-dire que $x>(1 / 2)$. Nous laissons au lecteur le soin de discuter le cas $x<(1 / 2)$ et de montrer en parliculier que dans cette éventualité les formules (39) subsistent encore. Mais alors :

$$
\Omega_{34}=i k
$$

Remarque. - On notera que dans les deux exemples qui précèdent les entiers $r_{1}$ et $r$ n'ont 
pu être déterminés, sans ambiguité, que moyennant un choix a priori d'un voisinage de $\Omega_{34}$ ou de $\Omega_{42}$ appartenant à $\hat{o}$. Mais lorsque se pìésente un problème concret, des considérations globales permettent toujours de lever cette indétermination d'ordre local.

22. Etudions maintenant la ligne libre $\mathrm{C}_{4}$ dans le voisinage de la singularité $\zeta=0$. Posons :

$$
z_{0}=x_{0}+i y_{0}
$$

il vient, en séparant le réel de l'imaginaire dans la deuxième formule (39) el pour de petites valeurs de $\zeta=\xi>0$ :

$x=x_{0}+\mathrm{R}_{3} \cos \pi x \xi+\ldots$,

$y=y_{0}+\mathrm{R}_{33} \sin \pi x \xi+\left(2 \mathrm{R}_{4} / 2 \%+1\right) \xi^{\alpha+(1 / 2)}+\ldots$,

les termes négligés étant de l'ordre de $O\left(\zeta^{2}\right)$, si $x>(1 / 2)$, et de l'ordre $O\left(\zeta^{(3 / 2)+a}\right)$ si $x<(1 / 2)$. Dans le premier cas, on voit que le contour $C$ présente une tangente continue dans le voisinage de la singularité; la ligne libre $\mathrm{C}_{4}$ se raccorde au premier ordre avec la ligne de suintement, d'équation :

$$
\left(x-x_{0}\right) \sin \pi x-\left(y-y_{0}\right) \cos \pi x=0 .
$$

Dans le second cas, $C_{4}$ présente au point $z_{34}=z_{0}$ une tangente verticale.

En tout état de cause, ces résultats se retrouvent à partir de l'équation approchée cartésienne de la ligne $C_{4}$ :

$$
\begin{aligned}
& y-y_{0}=\left(x-x_{0}\right) \lg \pi \% \\
& +\frac{2 \mathrm{R}_{4}}{(2 x+1)\left(\mathrm{R}_{2} \cos \pi x\right)^{a+(1 / 2)}}\left(x \cdots x_{0}\right) a+(1 / 2)
\end{aligned}
$$

qui permel d'ćtudier la courbure de $\mathrm{C}_{4}$ en $z_{34}$. On voit aisément que si $\alpha>(1 / 2)$, la courbure de la ligne libre en $z_{34}$ est infinie comme $x^{a-(3 / 2)}$; si $x<(1 / 2)$, cette courbure est infinie comme $x^{-2 a}$.

On étudierait, de même, $\mathrm{r}_{3}$ à partir de (39).

23. Passons à l'examen du cas singulier $x=(1 / 2)$. D'après ce qu'on a vu au chapilre II, nous arons encore (Cf. 34) :

$$
\mathrm{K}_{2}=-k \mathrm{R}_{4} ; \quad \mathrm{K}_{4}=\mathrm{R}_{4} i
$$

Les deux exposants caractéristiques sont maintenant : $\lambda_{1}=\lambda_{2}=0$, à des entiers près; nous laissons au lecteur le soin de vérifier que les entiers additifs sont nuls. Les formules (30) et (31), portées dans les relations $\left(18^{\prime}\right)$ el $(20)$, donment ici :

$\mathrm{K}^{\prime}{ }_{1}+K \mathrm{~K}_{3}^{\prime \prime}=0 ; \quad \mathrm{K}^{\prime \prime}{ }_{1}=0 ; \quad \mathrm{K}_{3}^{\prime}=\pi \mathrm{K}^{\prime \prime}{ }_{1}$

On a done :

$$
\mathrm{K}_{1}=-\mathrm{k} \mathrm{R} ; \quad \mathrm{K}_{3}=\pi \mathrm{R}_{4}+\mathrm{R} i,
$$

où $R$ est une constante réelle arbitraire. Par suite :

$$
\begin{aligned}
& \mathrm{F}=-k\left[\mathrm{R} u_{1}(\zeta)+\mathrm{R}_{4} \log \zeta u_{2}(\zeta)\right], \\
& Z=\left(\pi \mathrm{R}_{4}+\mathrm{R} i\right) u_{1}(\zeta)+\mathrm{R}_{4} i \log \zeta u_{2}(\zeta)
\end{aligned}
$$

La discussion s'achève aisément. Nous en résumons seulement l'essentiel. Dans le voisinage de $z=z_{34}$, le contour C forme une ligne à tangente continue; la ligne libre $\mathrm{C}_{4}$ présente, en son extrémité $z_{34}$, une courbure infinie. II serait bien facile de compléter notre étude par la discussion du signe des constantes $R$ et $R_{4}$.

\section{CHAPITRE IV}

\section{CONCLUSIONS}

24. Les exemples précédents nous paraissent de nature à mettre en Iumière la variété des singularités que peuvent présenter les fonctions $f(\zeta)$ et $z(\zeta)$ en un point-sommet de C. Il nous reste maintenant à dégager les conclusions que l'Ingénieur peut tirer de notre étude et à tracer la voie qu'il nous paraîtrait opportun de suivre pour perfectionner la théorie des écoulements plans, permanents, à potentiel, des eaux souterraines pesantes.

25. Nous avons déjà fait remarquer au paragraphe 4 que la construction analogique des écoulements étudies ici étail le seul moyen d'aborder, au bureau d'études, les problemes concrets; nous avons noté aussi que l'emploi de ces méthodes d'approximation était illégitime dans le voisinage d'une singularilé. Les résultats de notre mémoire apportent donc à l'Ingénieur un certain nombre de renseignements, à priori, qui peuvent être précieux. Nous savons désormais que, dans certains cas, la courbure de la ligne libre est nulle dans le voisinage d'une singularité, encore que la tangente à la ligne varie conlinûment. Il y aura alors lieu, pour l'Ingénieur, de déterminer, par un procédé ou par un autre, 
un arc de $\mathrm{C}_{4}$ pas trop éloigné de la singularité et de réunir cet arc au point singulier par une courbe de raccord dont les équations sont explicitées dans notre mémoire. Ceci exige la détermination numérique graphique des coefficients $\mathrm{R}_{j}(j=1,2,3,4)$. Nous espérons revenir sur cette technique dans une prochaine publication.

26. Il faut insister sur le fait que l'allure de $\mathrm{C}_{4}$ dans le voisinage d'une singularité peut dépendre des propriétés globales de l'écoulement. Tel est l'exemple du paragraphe 16. De nouvelles recherches paraissent nécessaires pour préciser l'influence des dimensions générales des éléments de $\mathrm{C}$, donnés, à priori, sur l'allure de la ligne libre.

27. Lorsque le nombre des points singuliers de $f(\zeta)$ ne dépasse pas 3 , ces fonctions sont hypergéométriques. Il est bien connu, en effet, que si une fonction $f(\zeta)$, holomorphe dans le demi-plan supérieur $\eta \geqslant 0$, présente sur l'axe réel trois singularités de type (9) et (10), elle est nécessairement solution d'une équation de Gauss. Cette remarque a été utilisée dans le grand traité de Poloubarinova-Kotchina, déjà tant de fois cité au cours de notre travail.

On voit donc que les séries hypergéométri- ques s'introduisent naturellement dans l'art de l'Ingénieur; ces belles fonctions trouvent ainsi une application nouvelle et importante dans la technique. Nous espérons revenir un jour sur ce point.

\section{BIBLIOGRAPHIE}

[1] Muskat. - « Flow of homogeneous fluids through porous media. 》 McGraw-Hill Book $\mathrm{Cy}$, New York and London, 1937.

[2] Poloubarinova-Kotchina. - « Théorie du mouvement des eaux souterraines $\gg$. Moscou, 1952, 676 pages (en russe). Voir spécialement le chapitre VII, pp. 304-326.

[3] J. Kravtchenko, G. Sauvage de Saint-Marc, M. BoreLr. - «Sur les singularités des écoulements plans, permanents des liquides en milieux poreux ». C.R. 1954, t. 238, pp. 204-211.

[4.] M. Borelr. - " Contribution à l'étude des milieux poreux », thèse d'ingénieur + docteur, soutenue devant la Faculté des Sciences de Grenoble, janvier 1954. A paraître dans la collection scientifique et technique du Ministère de l'Air.

[5] J. Kravtchenko, G. Sauvage de Saint-Marc, M. Borel. - «Sur les singularités des fonctions analytiques définies par certaines conditions aux limites ». Article à paraître.

[6] A. CaSagrande. - "Seepage through dams 》. New England water works Association 1937, p. 303.

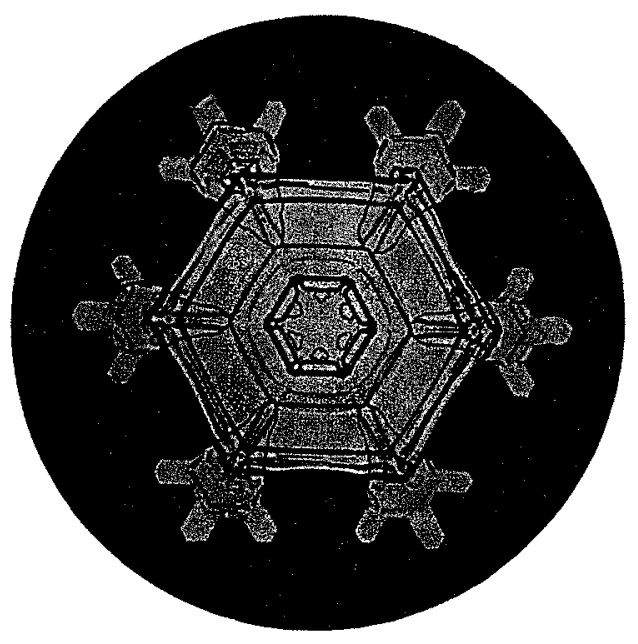

